\title{
Bifurcation analysis of a periodically forced relaxation oscillator: Differential model versus phase-resetting map
}

\author{
H. Croisier, ${ }^{1 *}$ M. R. Guevara, ${ }^{2}$ and P. C. Dauby ${ }^{1}$ \\ ${ }^{1}$ Institut de Physique B5a, Université de Liège, Allée du 6 Août 17, 4000 Liège, Belgium \\ ${ }^{2}$ Department of Physiology and Centre for Nonlinear Dynamics in Physiology and Medicine, \\ McGill University, 3655 Sir William Osler Promenade, Montreal, Quebec, Canada H3G 1 Y6
}

(Received 20 June 2008; published 20 January 2009)

\begin{abstract}
We compare the dynamics of the periodically forced FitzHugh-Nagumo oscillator in its relaxation regime to that of a one-dimensional discrete map of the circle derived from the phase-resetting response of this oscillator (the "phase-resetting map"). The forcing is a periodic train of Gaussian-shaped pulses, with the width of the pulses much shorter than the intrinsic period of the oscillator. Using numerical continuation techniques, we compute bifurcation diagrams for the periodic solutions of the full differential equations, with the stimulation period being the bifurcation parameter. The period-1 solutions, which belong either to isolated loops or to an everywhere-unstable branch in the bifurcation diagram at sufficiently small stimulation amplitudes, merge together to form a single branch at larger stimulation amplitudes. As a consequence of the fast-slow nature of the oscillator, this merging occurs at virtually the same stimulation amplitude for all the period-1 loops. Again using continuation, we show that this stimulation amplitude corresponds, in the circle map, to a change of topological degree from one to zero. We explain the origin of this coincidence, and also discuss the translational symmetry properties of the bifurcation diagram.
\end{abstract}

DOI: 10.1103/PhysRevE.79.016209

PACS number(s): 05.45.Xt, 87.19.1n, 87.19.Hh

\section{INTRODUCTION}

Biological systems that oscillate can often be modeled as relaxation oscillators, in which the state point of the system moves relatively slowly within two well-separated regions of the phase space (the slow or "relaxation" phases of the oscillation), with much faster jumps between these two regions $[1,2]$. The FitzHugh-Nagumo (FHN) model is a simple twovariable model of an excitable cell, in which excitability is intimately linked with the fast-slow nature of the trajectories [3]. Indeed, it can be easily turned into a relaxation oscillator by changing a single parameter away from its nominal value [3]. This is not at all surprising, given that the original excitable FHN system came from the van der Pol oscillator, modified so as to remove the limit-cycle oscillation but maintain excitability [4]: hence the original name given to these equations by FitzHugh, the Bonhoeffer-van der Pol equations [3].

Biological oscillators are in turn themselves typically subject to external periodic forcing [5]. There have been many studies on the periodically forced FHN oscillator (e.g., [6-11]), as well as on the periodically forced van der Pol oscillator (e.g., see [12] for a review; $[2,13,14]$ ). These have yielded a plethora of rhythms, including periodic ("phaselocked"), quasiperiodic, and chaotic rhythms. Similar behaviors are seen in low-dimensional nonrelaxation oscillators [13-37], and in low-dimensional fast-slow excitable systems $[10,11,30,38-48]$.

In order to gain insight into the extremely complicated response to periodic stimulation, the usual practice of carrying out direct numerical integration of the full equations is insufficient. Two additional approaches have been employed in the literature. The first one uses numerical continuation

\footnotetext{
*hcroisier@ulg.ac.be
}

methods, coupled with algorithms to identify bifurcations, to study the periodic solutions of the full differential equations (or, equivalently, to study the fixed points of the stroboscopic maps of the system). While such methods have been used many times to study forced oscillators in their nonrelaxation regime [17,18,21-25,29,31-33,35-37], and, to a lesser extent, forced fast-slow excitable systems $[39,48]$, they have been applied only rarely to forced oscillators in the relaxation regime (e.g., [13]).

The second approach involves some degree of approximation, in that one reduces consideration of the forced twodimensional system to analysis of a one-dimensional discrete map, for which the numerical computations are easier, and for which one can occasionally even obtain analytic solutions. This has been carried out in two ways. In the first way, one assumes the singular limit, so that the motion of the unforced oscillator is essentially one dimensional, with the state point of the system moving on the two stable sheets of the critical manifold except for instantaneous jumps between these two sheets $[2,14,44-46,49-51]$. In the second way, one first characterizes the phase-resetting response of the oscillator to a single stimulus pulse delivered systematically at various phases throughout its cycle. Then, assuming that there is a sufficiently fast return of the trajectory back to the limit cycle, one derives a one-dimensional circle map (the phaseresetting map) that can then be iterated to predict the response to periodic stimulation (e.g., [6,10,19,52-55]). A onedimensional return map has sometimes also been obtained by measuring the phases of the cycle at which successive stimuli arrive during periodic stimulation (e.g., $[8,54,56]$ ). It is known that an important topological feature of these one-dimensional circle maps, namely, their topological degree, changes with stimulation amplitude (e.g., $[9,19,26,54,55,57-59])$ and that this topological degree constrains the classes of rhythms that can be seen (e.g., [60]). 
In this paper, we show that the topological degree of the phase-resetting map of a relaxation oscillator changes from one to zero at the same stimulation amplitude as that at which the period-1 loops in the bifurcation diagram of the full differential equations merge with an everywhereunstable period- 1 branch. We explain this coincidence by the fact that the bifurcation diagram of any circle map where the bifurcation parameter (in our case, the stimulation period) appears only in an additive fashion has its period- 1 fixed points belonging to isolated loops when the topological degree of the map is one, while these fixed points belong to a unique branch when the topological degree of the map is zero. Therefore, if the phase-resetting map is a good approximation of the full differential equations, it must change topological degree for the same stimulation amplitude as that at which the merging of period- 1 solution branches occurs in the bifurcation diagram of the original equations. To our knowledge, this is the first time that this property of circle maps has been underscored. We also discuss the extent to which the translational symmetry that characterizes the bifurcation diagram of circle maps $[19,61]$ holds in the bifurcation diagram of the differential equations.

The paper is organized as follows. In Sec. II, we perform a continuation analysis of the differential model, which is a forced FHN oscillator in its relaxation regime, using the stimulation period as bifurcation parameter. We study how the bifurcation diagram evolves as the stimulation amplitude is raised. In particular, we note that the merging of the period-1 loops with the everywhere-unstable branch of the same period occurs at virtually the same amplitude for all loops. In Sec. III, we investigate how well this evolution is accounted for by the phase-resetting map. We use a graphical method to obtain the period-1 fixed points of the map, which allows us to deduce the topological property of circle maps announced above. We then take advantage of the translational invariance of the bifurcation diagram of circle maps $[19,61]$ in order to compare the period- $M$ bifurcation points of the map to those of the differential equations. Finally, in Sec. IV, we put our results into perspective with prior related works.

\section{DIFFERENTIAL MODEL OF THE FORCED RELAXATION OSCILLATOR}

\section{A. Equations and numerical methods}

We study the periodically forced FHN oscillator:

$$
\begin{gathered}
\frac{d u}{d t}=A u(1-u)\left(u-u_{s}\right)-v+I(t), \\
\frac{d v}{d t}=\varepsilon(u-c v-d),
\end{gathered}
$$

where $A=1, u_{s}=0.2, c=0.4, d=0.2$, and $\varepsilon=0.005$. The parameter values are such that the unforced system $[I(t)=0]$ possesses a globally attracting limit cycle, enclosing an unstable node. The period of the limit cycle is $T_{0}=165.191$. If one thinks of Eq. (1) as being a reduced form of the Hodgkin-Huxley model, then the variable $u$ corresponds to the electrical potential across the cell membrane while the variable $v$ is a slow "gating" variable. The difference of time scale between the two variables, which is characteristic of relaxation oscillations, is produced by the smallness of $\varepsilon$.

The periodic stimulation $I(t)$ is a train of Gaussian-shaped pulses:

$$
I(t)=I_{0} \sum_{j=-\infty}^{\infty} \exp \left[-(t-j T)^{2} / \sigma^{2}\right],
$$

where $I_{0}$ is the stimulation amplitude, $T$ is the stimulation period, and $\sigma=1$. This choice of $\sigma \ll T_{0}$ guarantees that the stimulus is effectively "on" during a time much shorter than the intrinsic period of the oscillator, since the Gaussian function, although never zero, is rapidly decreasing.

We used the method described in [48] in order to follow periodic solutions of (1)-(2)with AUTO97 continuation software [62] (see also the discussion in Sec. IV below).

\section{B. Theoretical background}

Before describing our numerical results, let us summarize a few theoretical results about periodically forced planar oscillators, under the hypothesis that the unforced oscillator is described by a globally attracting limit cycle of period $T_{0}$ enclosing an unstable fixed point (see also [23,31,32,63]). The extended phase space of the forced oscillator is $\mathbb{R}^{2}$ $\times \mathrm{S}^{1}$, since the unforced oscillator is two-dimensional (2D) and since the forcing is a periodic function of time unaffected by the oscillator, allowing the time to be considered modulo the (normalized) stimulation period $T / T_{0}$.

At zero stimulation amplitude, and for any value of $T / T_{0}$, the extended phase space contains a globally attracting invariant two-torus, given by the product of the globally attracting limit cycle of the unforced system by $\mathrm{S}^{1}$, which describes the forcing, and an unstable limit cycle of period 1 , enclosed within the torus, given by the product of the unstable fixed point of the unforced system by $\mathrm{S}^{1}$. The globally attracting character of the invariant torus means that all attractors must lie on the torus, although the torus may not be an attractor itself (see, e.g., [64], Chap. 1).

For sufficiently small stimulation amplitude, both the invariant torus and the unstable limit cycle enclosed by the torus are guaranteed to persist, because they are "normally hyperbolic" invariant manifolds at zero stimulation amplitude $[65,66]$. (An invariant manifold is normally hyperbolic if, under the dynamics linearized about the invariant manifold, the growth rate of vectors transverse to the manifold dominates the growth rate of vectors tangent to the manifold [66].) The normal hyperbolicity of the torus is a consequence of the hyperbolicity of the attracting limit cycle of the unforced equations, while the normal hyperbolicity of the unstable limit cycle enclosed by the torus is a consequence of the hyperbolicity of the repelling fixed point of the unforced equations. With the exception of the unstable limit cycle enclosed by the torus, no hyperbolic solution can exist outside of the globally attracting torus at small forcing amplitude, because it would then have to exist at zero amplitude, while by assumption no such orbit exists for $I_{0}=0$. Hence, for $I_{0}$ 


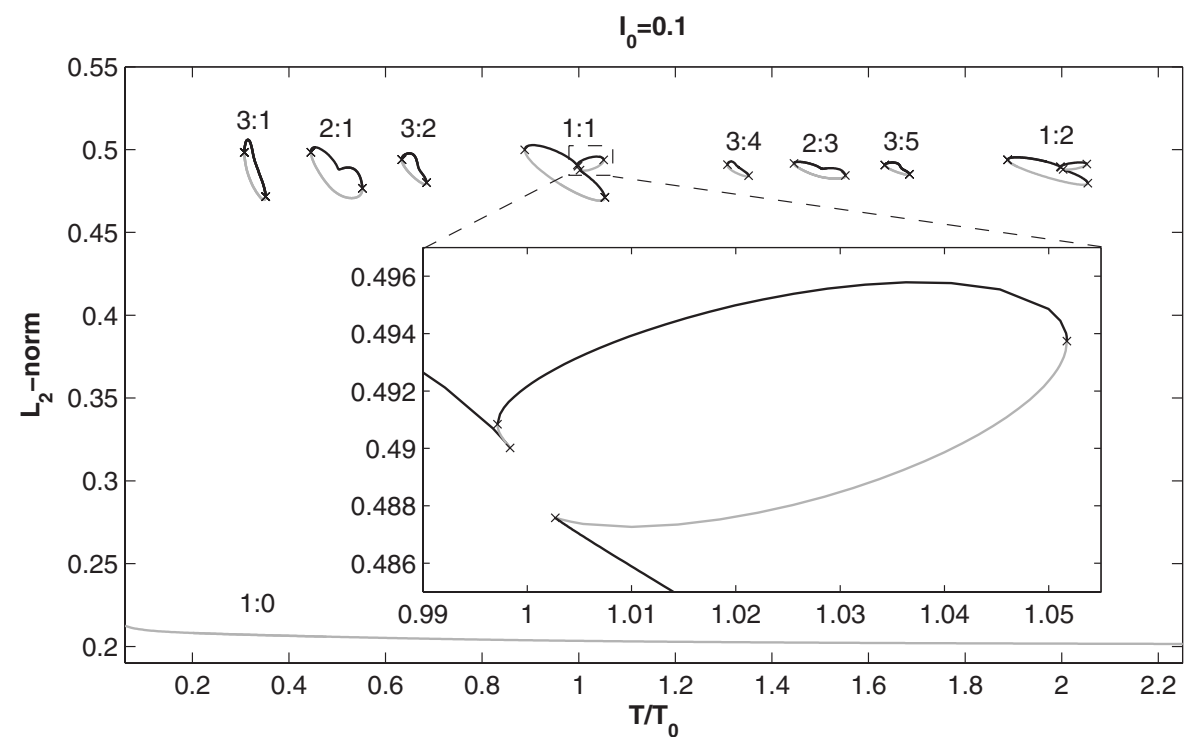

FIG. 1. Bifurcation diagram computed with AUTO for $I_{0}=0.1$. Black curves indicate stable solutions; gray curves indicate unstable solutions. The only bifurcations present are saddle-nodes $(\times)$. The zoom on part of the $1: 1$ loop shows two of the three pairs of SNs on that loop, which give rise to $1: 1 \leftrightarrow 1: 1$ bistability $(1: 1$ self-bistability) in two ranges of $T / T_{0}$.

sufficiently small, the asymptotic dynamics is essentially restricted to the invariant torus, and this constrains substantially the types of behavior that can occur. In particular, the existence of a globally attracting invariant torus for the differential equations is equivalent to the existence of a globally attracting invariant circle for the "stroboscopic maps" of the system (the 2D discrete maps obtained by stroboscopically sampling the flow at time intervals equal to the forcing period). This implies that the study of the forced oscillator essentially reduces to that of a family of discrete maps of the circle. These circle maps are, moreover, invertible since the stroboscopic maps of differential flows are invertible maps of the plane. Many important properties have been proved for such invertible circle maps (e.g., [67-69]), some of which we enunciate now in terms of the invariant torus of the differential equations.

A rotation number can be associated with every solution on the torus. Geometrically, it corresponds to the average number of times the solution winds around the meridian of the torus per forcing period. When the rotation number is rational, the solution is periodic; when it is irrational, the solution is quasiperiodic, with the trajectory densely covering the torus. The rotation number is unique for a given value of $\left(T / T_{0}, I_{0}\right)$, which implies that the coexistence of periodic solutions of different period, as well as the coexistence of periodic and quasiperiodic motions, is forbidden on the torus (this would indeed imply the intersection of the trajectories). A stable periodic solution never exists alone on the torus, but is always paired with an unstable solution of the same period, with the stable solution being a nodal limit cycle, and the unstable one being a saddle limit cycle. The region in the $\left(T / T_{0}, I_{0}\right)$ parameter plane where a periodic solution with period $M T$ and rotation number $N / M$ exists is called an $N / M$ Arnol'd tongue, or $N / M$ resonance horn, or $M: N$ phaselocking zone ( $M, N$ integers). Each $N / M$ Arnol'd tongue originates from the point $T / T_{0}=N / M$ on the $T / T_{0}$ axis and generically opens up into the upper half of the parameter plane as a "wedge" (i.e., the two sides of the tongue are not tangent to one another at zero amplitude), bounded by saddle-node bifurcation curves.
At larger stimulation amplitudes, the invariant torus is no longer guaranteed to persist, and indeed typically breaks up. The aforementioned results then no longer hold and one has to revert to studying the full differential equations, or the associated stroboscopic maps of the plane. Although some general results do exist about the latter (e.g., [32]), the dynamics of such systems is much less constrained than that of circle maps, so that numerical continuation is often needed to study the details of the wealth of phenomena that can occur.

\section{Results}

We have computed bifurcation diagrams with the stimulation period $T$ as bifurcation parameter, the stimulation amplitude $I_{0}$ being fixed, restricting our analysis to periodic solutions of period $P=M T$ (or period- $M$ solutions) with $M$ $\leqslant 3$. The stimulation period $T$ was kept above $T_{\min } \simeq 10 \sigma$, in order to avoid substantial overlap of successive stimuli, and below some $T_{\max }$ which depends on the bifurcation diagram, but chosen large enough so that the 1:2 rhythm is computed in each case $\left(2.25 T_{0}<T_{\max }<3 T_{0}\right)$. In all the bifurcation diagrams that follow, black curves represent stable solutions, gray curves represent unstable solutions, black crosses are saddle-node (SN) bifurcations, and purple circles are perioddoubling (PD) bifurcations. The normalized stimulation period $T / T_{0}$ is plotted along the abscissa. The $L_{2}$-norm of the solutions, that is, $\left\{(1 / P) \int_{0}^{P}\left[u^{2}(t)+v^{2}(t)\right] d t\right\}^{1 / 2}$, where $P$ is the period of the solution, is plotted along the ordinate. The " $M: N$ " labels indicate the phase-locked rhythms to which the period- $M$ solutions correspond ("phase-locked rhythm" is used in this paper indifferently from "periodic solution," with no implication about the stability of the solution). The way these labels are assigned is discussed at the end of this section. We now describe the evolution of the bifurcation diagram as $I_{0}$ is increased.

For very small stimulation amplitudes, e.g., $I_{0}=0.1$ (Fig. 1), all stable periodic solutions belong to isolated closed loops in the bifurcation diagram. The only bifurcation points on these loops are saddle-nodes, which is consistent with the persistence of the invariant torus. Most loops in Fig. 1 con- 

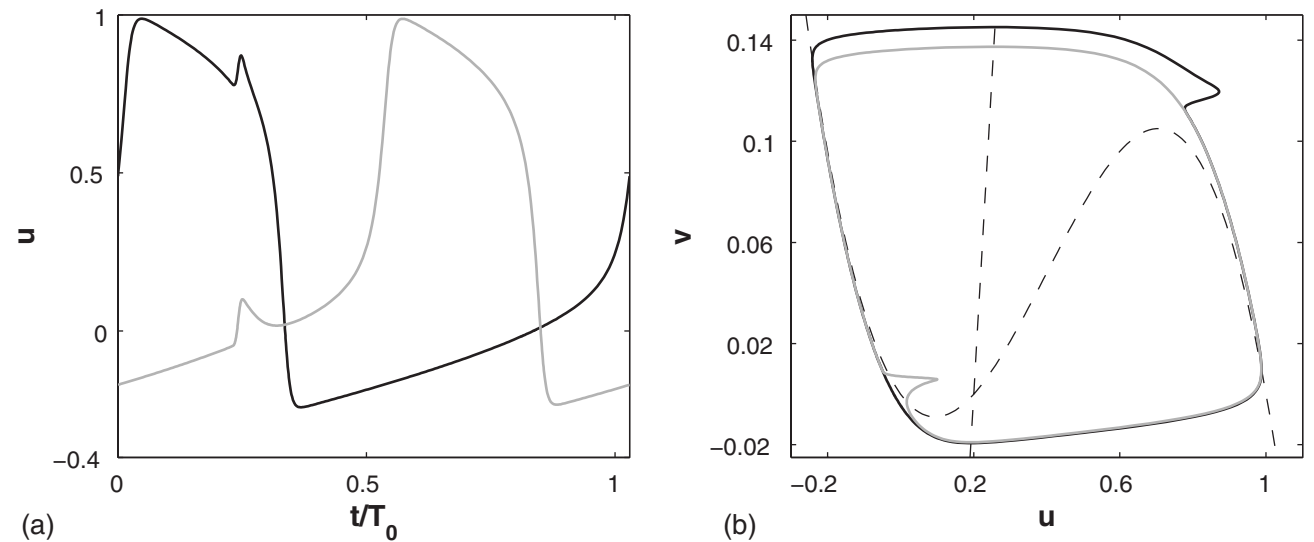

FIG. 2. The pair of bistable 1:1 rhythms existing at $I_{0}=0.1, T / T_{0}=1.03$. (a) $u(t)$ plotted over one period. (b) projection of the solutions on the $(u, v)$ phase plane (solid curves). The trajectories are traveled counterclockwise. Dashed curves give the nullclines of the unforced system.

tain only two saddle-nodes, where the stable-unstable pairs of solutions meet, and which define their boundaries with respect to $T / T_{0}$ (and thus give the edges of the Arnol'd tongue in cross section at that $I_{0}$ ). The period-1 loops, however, contain three pairs of saddle-nodes, which gives rise to regions of bistability. The zoom in Fig. 1, for instance, shows that there are two ranges of $T / T_{0}$ over which there is coexistence of two stable $1: 1$ rhythms $\left(0.9971<T / T_{0}<0.9983\right.$ and $\left.1.003<T / T_{0}<1.052\right)$, which we term $1: 1$ "selfbistability." Figure 2 shows one of these pairs of bistable 1:1 rhythms. Continuation of the period-1 saddle-nodes in the two-parameter $\left(T / T_{0}, I_{0}\right)$ plane shows that the bistability exists all the way down to zero amplitude (Fig. 6). This does not contradict the persistence of the globally attracting torus mentioned earlier since these solutions have the same period. In fact, the occurrence of such self-bistability at arbitrarily small stimulation amplitudes has been shown to be a generic feature of periodically forced planar oscillators (see [70] and the discussion in Sec. IV D). The regions in the twoparameter plane which are bounded by the "secondary" SN curves in Fig. 6 (dashed and dotted curves) have been termed Arnol'd flames [63,70].

In addition to the isolated loops, the bifurcation diagram at small $I_{0}$ contains an everywhere-unstable period- 1 branch (bottom branch in Fig. 1). This branch reflects the persistence at finite $I_{0}$ of the unstable limit cycle existing in the extended phase space at zero amplitude. Projection of solutions from this branch on the $(u, v)$ phase plane shows that the state point indeed remains in the vicinity of the unstable node of the unforced system, passing closer and closer to it as the stimulation period increases (Fig. 3). These solutions are reminiscent of canards, in that a segment of the trajectory remains close to the middle branch of the $u$-nullcline, which constitutes the unstable part of the critical manifold of the unforced equations (the critical manifold of a fast-slow system is the nullcline associated with the fast variable; see, e.g., [71]). The standard rotation number is not defined for these solutions since, for each given value of $T / T_{0}$, the solution does not lie on the invariant torus but constitutes the unstable limit cycle enclosed by the torus. However, if one uses the "physiological" definition that an $M: N$ rhythm is a period- $M$ response in which $N$ action potentials are elicited, these solutions have to be called 1:0 rhythms, because the period-1 response is of too small an amplitude to be considered an action potential (AP). Indeed, in the FHN model, an AP is notably characterized by the state point visiting the vicinity of the right branch of the $u$-nullcline [as is the case, e.g., in the phase-plane trajectories in Fig. 2(b)].

Another characteristic of Fig. 1 is the approximate translational invariance of the bifurcation diagram. If the loops to the right of the $1: 1$ rhythm are shifted left by an amount $T / T_{0}=1$, then each $M: N$ loop for $T / T_{0}>1.2$ is paired with an $M: N-M$ loop for $T / T_{0}<1.2$, and the locations of the saddle-nodes on each pair of loops virtually coincide (Fig. 4). We shall return to consider this symmetry further later on (Sec. III B 5).

At a higher value of $I_{0}$, pairs of period-doubling points appear on the isolated loops. They are present, e.g., for $I_{0}$ $=0.133$ (Fig. 5). In the ranges of $T / T_{0}$ where a period-

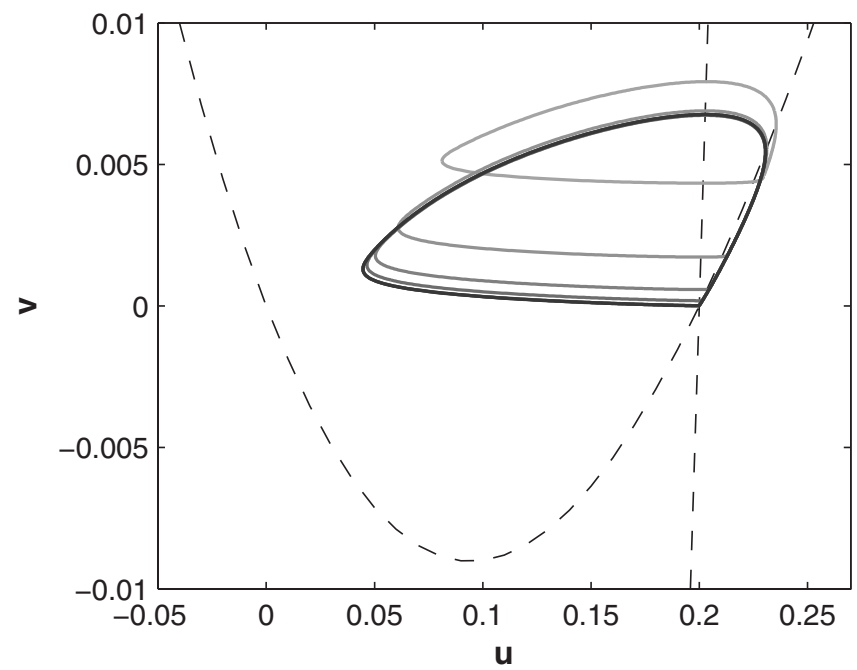

FIG. 3. Projections onto the $(u, v)$ phase plane of solutions belonging to the unstable 1:0 branch for $I_{0}=0.1$, obtained using AUTO. The unstable fixed point of the unforced system lies at the intersection of the nullclines (dashed curves). $T / T_{0}$ varies from 0.234 (lightest gray curve) to 2.46 (black curve). 


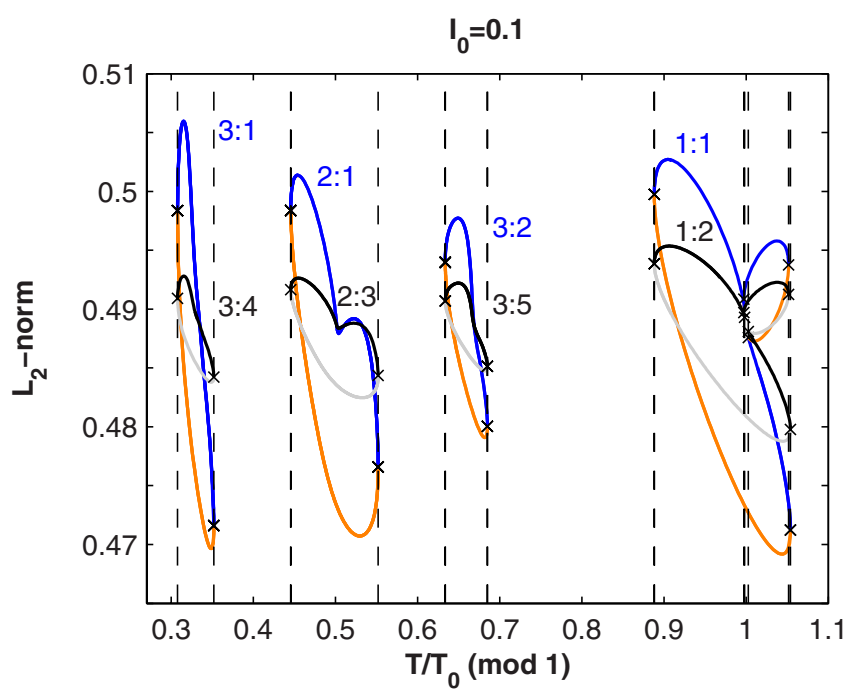

FIG. 4. (Color online) Illustration of the approximate translational symmetry of the bifurcation diagram for $I_{0}=0.1$. The loops to the right of the $1: 1$ rhythm are shifted left by an amount $T / T_{0}=1$. For the unshifted loops, blue indicates stable solutions, orange indicates unstable solutions. The locations of the SNs for the shifted loops are highlighted by vertical dashed lines, to allow comparison with the corresponding bifurcations for the unshifted loops.

doubled solution is born, the invariant torus can no longer exist. Indeed, for a given value of $T / T_{0}$, the period-doubled solution cannot lie on the invariant torus because it would intersect the periodic solution from which it originates. Neither can it lie outside of this torus because this would imply a crossing of the invariant torus by the period-doubled solution.
One of the points from each PD pair is very close to the rightmost SN of each loop (e.g., at point $C$ in Fig. 5), so that the two different bifurcations look like they are occurring at the same point in the figure. On some loops, the right PD point was not even identified by AUTO. This is because the continuation step used in these computations was not small enough to detect the two consecutive bifurcations (SN-PD). We have performed a two-parameter continuation of the left PD point for some loops and in each case, the PD bifurcation curve leads to the right PD point, as shown in Fig. 6 for the 1:1 loop. Figure 6 also shows that the proximity of the rightmost SN and PD points actually persists over a large range of parameters values, since the bottom part of the PD branch (thick purple curve) is indistinguishable from the nearest SN branch (solid black curve). The two bifurcation curves become more separated from each other as larger values of $\varepsilon$ are used, and so we believe that their close proximity in Fig. 6 is related to the nearly singular nature of the system $[\varepsilon$ $\ll 1$ in Eq. (1)]. The fact that the two bifurcation curves extend greatly to the right (they actually extend further than the right limit of Fig. 6), in a quasihorizontal manner, is also due to the smallness of $\varepsilon$.

The two PD points of each pair (e.g., $A$ and $C$ in Fig. 5) are connected by a branch of period- 2 solutions. However, for the sake of clarity, we show in Fig. 5 the period- 2 branch only for the 1:1 loop, and use a different color scheme to indicate its stability (blue for stable solutions, orange for unstable solutions) to distinguish this branch from the period-1 branch. This period-2 branch is unstable for most of the range of $T / T_{0}$ values over which it exists, as the dominant orange (gray) color indicates. A stable 2:2 rhythm is, however, present in the tiny region between point $A$ (PD of period-1 solution) and point $B$ (PD of period-2 solution). The

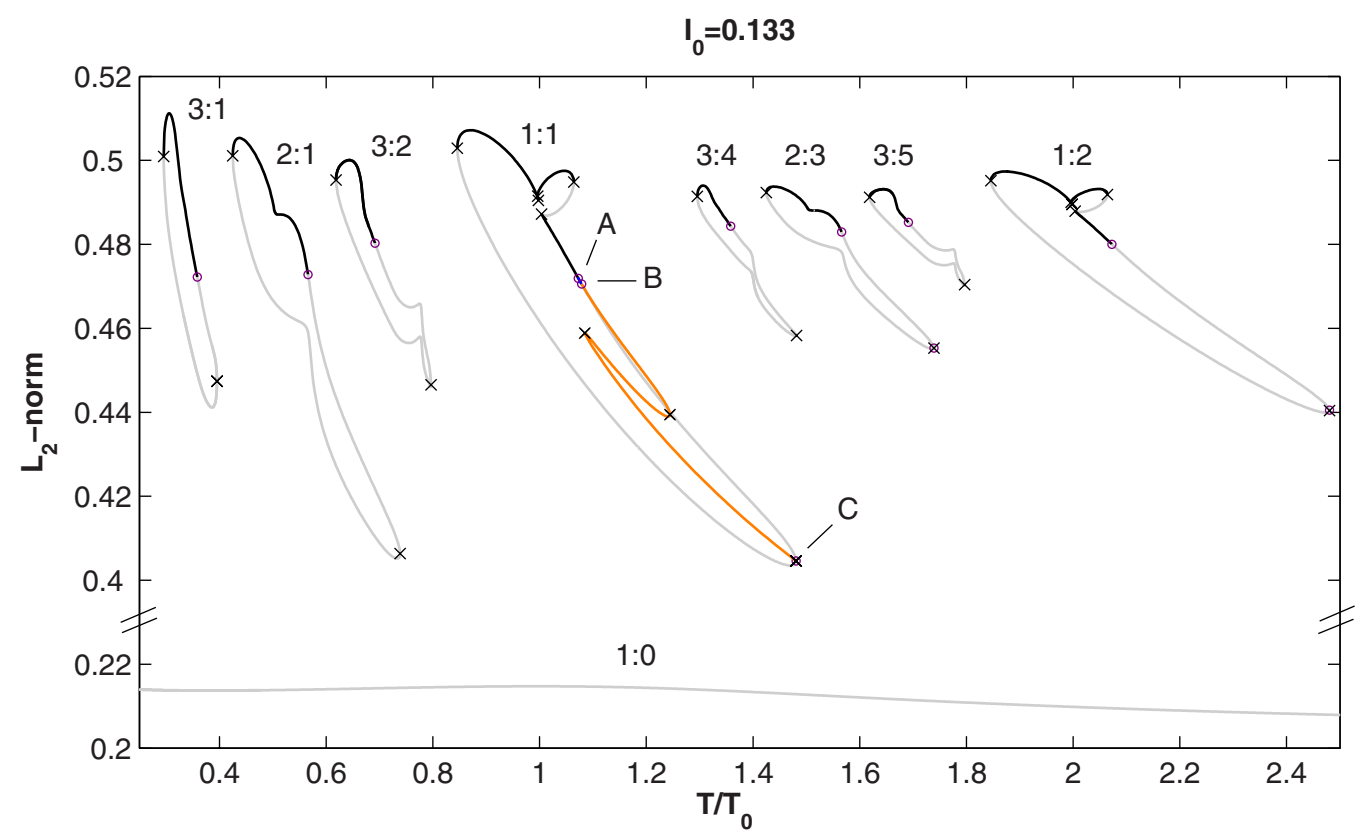

FIG. 5. (Color online) Bifurcation diagram for $I_{0}=0.133$. Pairs of PD points $(\bigcirc)$ have appeared on the loops, with the rightmost PD point of each pair being very close to a SN $(\times)$, as at point $C$. The rightmost PD point has sometimes been missed by the computation (cf. text). The period-2 branch that connects each pair of PD points is shown only for the 1:1 loop. It is stable in the tiny region between point $A$ and point $B$ (blue color) and unstable virtually everywhere between point $B$ and point $C$ (orange color). The PD point $A$ belongs to the period-1 branch while the PD point $B$ belongs to the period-2 branch. Note the $y$-axis break. 


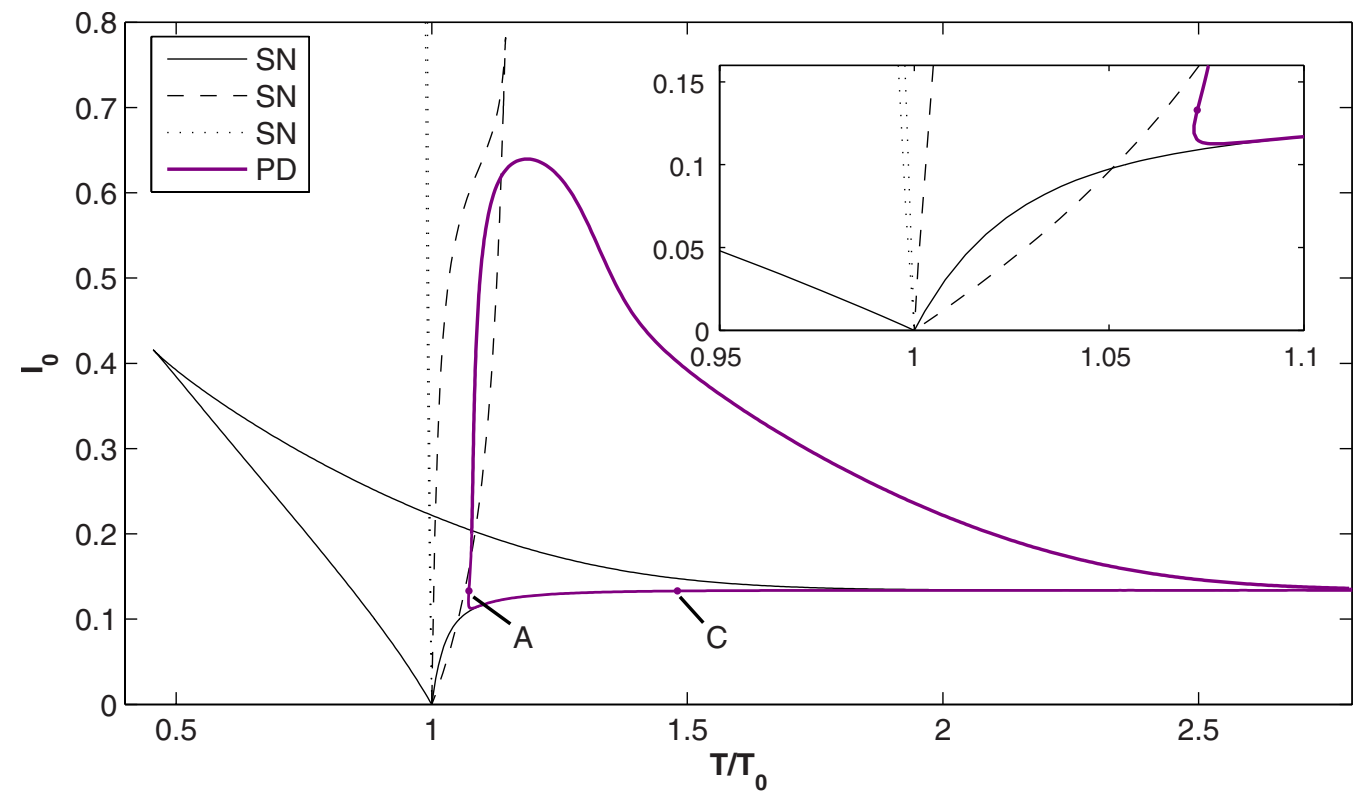

FIG. 6. (Color online) Loci of the three pairs of SNs (thin solid, dashed, and dotted black curves) and of the pair of PDs (thick purple curve) on the 1:1 loop. The zoom shown in the inset allows the two SNs belonging to the narrowest locus (dotted curve) to be distinguished from each other. Points $A$ and $C$ are those from Fig. 5.

two PD points $A$ and $B$ might thus be the first two members of a supercritical period-doubling cascade that produces period-doubled phase-locking zones within the Arnol'd tongues, and which can even progress to chaotic dynamics (e.g., $[17,29])$.

If the amplitude is raised just a little further, a spectacular change occurs in the bifurcation diagram: the period-1 loops merge with the everywhere-unstable period- 1 branch, and the period-2 loops merge with period-2 branches that have grown out of the everywhere-unstable period-1 branch via the birth of pairs of PD points. For instance, for $I_{0}=0.135$ in Fig. 7, both the 1:1 loop and the 1:2 loop have merged with the 1:0 branch, the $2: 3$ loop has merged with a period-2 branch emanating from the period- 1 branch, and the $2: 1$ loop is close to undergoing the same process as the 2:3 loop with another period- 2 branch that has grown below the 2:1 loop.

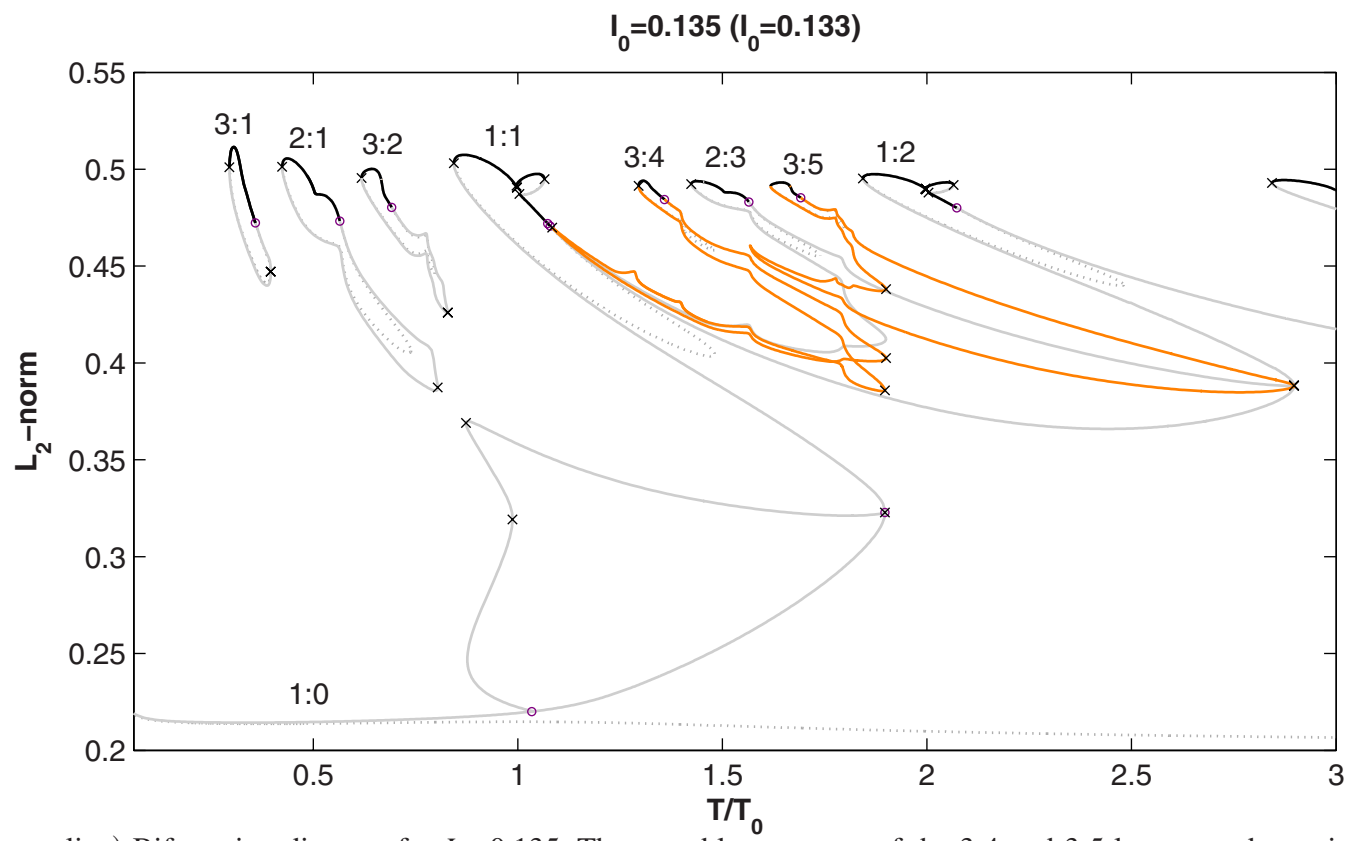

FIG. 7. (Color online) Bifurcation diagram for $I_{0}=0.135$. The unstable segments of the $3: 4$ and $3: 5$ loops are drawn in orange, in order to facilitate their identification. The dotted curves in the background repeat the bifurcation diagram for $I_{0}=0.133$ (disregarding stability), in order to underline the huge qualitative change that has occurred. Note how the $2: 1$ loop is about to merge with the period-2 branch immediately below it. 

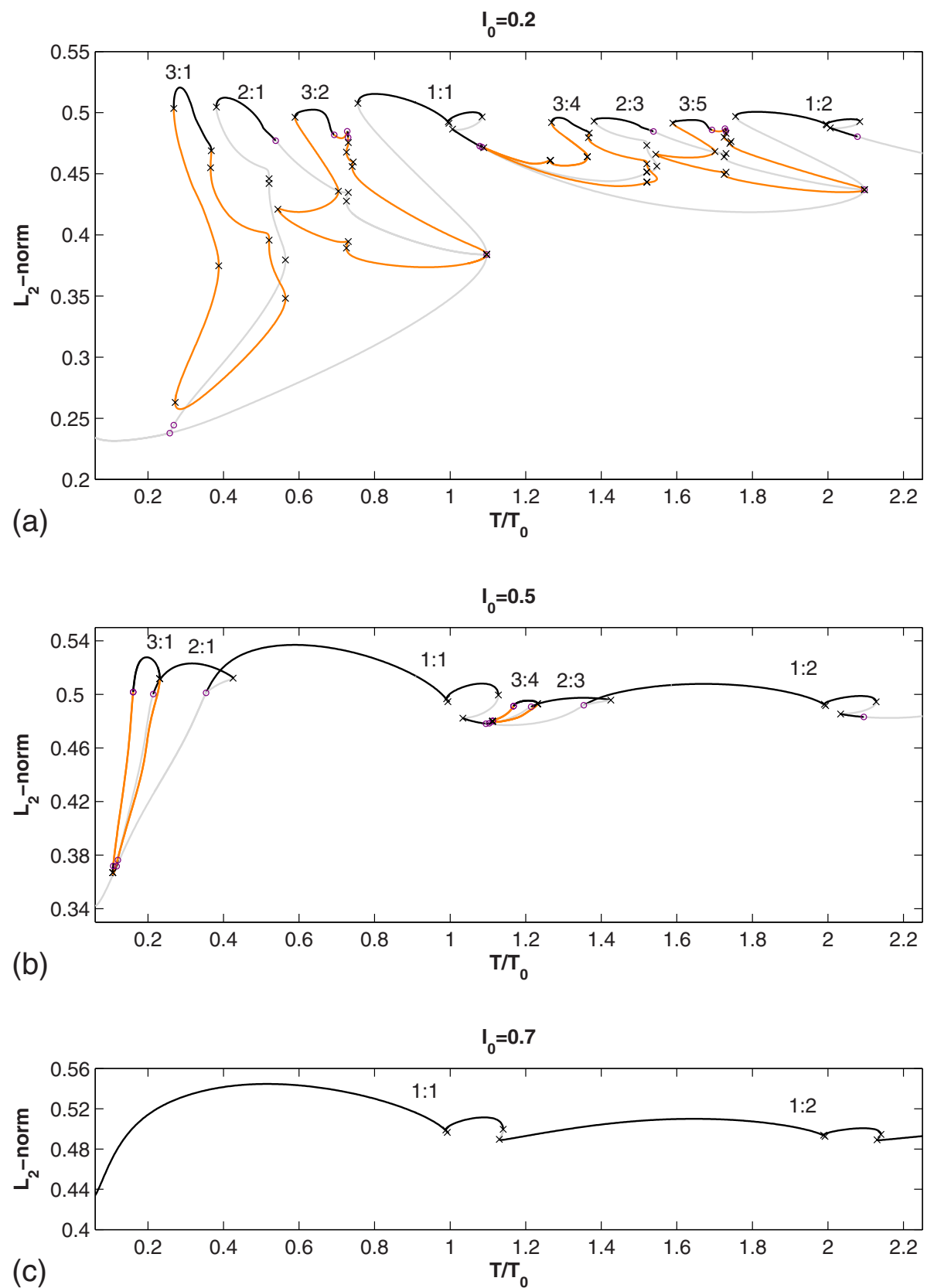

FIG. 8. (Color online) Bifurcation diagrams for $I_{0}=0.2,0.5$, and 0.7 . The unstable portions of the period-3 loops are drawn in orange.
We were not able to determine numerically which of the period-1 loops merges first with the unstable period-1 branch: we find that all merge for $I_{0}=I_{\text {th }}=0.1338 \pm 0.0001$. We are convinced that this very delicate behavior is related again to the nearly singular nature of the system, since the successive mergings can be distinguished if a larger value of $\varepsilon$ is used (e.g., using $\varepsilon=0.02$ and $\sigma=0.25$ ). It should be noted that the $3: 4$ and $3: 5$ solutions, like the $3: 1$ and $3: 2$ solutions, still form closed loops for $I_{0}>I_{\mathrm{th}}$. But because the unstable parts of these loops pass very close to the period-1 and period- 2 branches, this is not easy to realize at first sight (we have used orange in Fig. 7 to highlight the unstable segments of the period-3 loops). The crossings of the 3:4 and 3:5 loops with the 2:3 loop are only apparent (two different solutions can have the same $L_{2}$-norm). The rightmost $\mathrm{SN}$ on the 3:5 loop is very close to a $\mathrm{SN}$ on the period- 1 branch, and remains so over a finite range of stimulation amplitude. This characteristic is yet again due to the use of a small value of $\varepsilon$. Finally, the dotted gray curves in the background of Fig. 7 superimpose the bifurcation diagram for $I_{0}=0.133$ (disregarding stability), in order to underscore the huge change that has occurred in the qualitative picture following the minute change of amplitude from $I_{0}=0.133$ to 0.135 .

For $I_{0}=0.2$ [Fig. 8(a)], the 2:1 loop has merged with the period-2 branch below it. The 3:2 loop, in a fashion similar to the 3:5 loop, now has its rightmost $\mathrm{SN}$ virtually touching the period-1 branch.

For $I_{0}=0.5$ [Fig. 8(b)], we no longer find stable $3: 2$ and $3: 5$ rhythms. Instead, $2: 1 \leftrightarrow 1: 1$ bistability and $2: 3 \leftrightarrow 1: 2$ bistability are seen. $3: 1 \leftrightarrow 2: 1$ bistability and $3: 4 \leftrightarrow 2: 3$ bistability are also present.

For $I_{0}=0.7$ [Fig. 8(c)], only the period-1 branch remains. All the period-doubled branches have disappeared through collision and annihilation of the PD points on each branch. 
All the loops have also disappeared, and it is likely that the period-1 branch is truly the only periodic solution left, since the external forcing is expected to dominate the dynamics of the system at very large stimulation amplitudes, as in several other models of periodically forced oscillators $[20,23,29-34,63]$. One of the two pairs of saddle-nodes still present on the 1:1 branch for $I_{0}=0.7$ (the rightmost pair) disappears at slightly larger stimulation amplitudes $\left(I_{0}\right.$ $=0.782$ ), as can be seen from the closing of the dashed curve in Fig. 6. The remaining pair (dotted curve in Fig. 6) disappears only at a huge stimulation amplitude $\left(I_{0}=155\right)$.

Before closing this section, let us discuss briefly how we have labeled the $M: N$ rhythms in the bifurcation diagrams. With the exception of the everywhere-unstable period-1 branch existing at small $I_{0}$, we have labeled only the stable portions of branches. At small stimulation amplitudes (Fig. 1), the distinction between the different stable rhythms is unequivocal since each loop in the bifurcation diagram corresponds to a single Arnol'd tongue, of which it constitutes the "cross section" at that amplitude. The rhythm is " $M: N$ " if the corresponding Arnol'd tongue originates at $T / T_{0}$ $=N / M$. At intermediate and large stimulation amplitudes, we have assumed that if a stable segment corresponds to an $M: N$ rhythm at small stimulation amplitudes, it remains an $M: N$ rhythm as the amplitude is raised. This seems a reasonable assumption as long as different stable rhythms of the same period (i.e., an $M: N$ rhythm and an $M: N+1$ rhythm) are isolated from each other (Fig. 5), or belong to the same branch but are separated by a large unstable region [Fig. 7 and Fig. 8(a)], since the difference in $T / T_{0}$ guarantees to some extent that the solutions "look" different. However, this assumption becomes questionable at large amplitudes, where the unstable segments between stable portions of branches are reduced to very small ranges of $T / T_{0}$ [Fig. 8(c)]. The labeling in Fig. 8(c) should therefore be considered as approximate, but this looseness does not matter for the points made in this paper.

\section{PHASE-RESETTING MAP AND COMPARISON WITH THE ORDINARY DIFFERENTIAL EQUATION}

\section{A. Prerequisite}

\section{Definitions}

Consider an ordinary differential equation (ODE) possessing a stable limit cycle of period $T_{0}$ and suppose that the state point lies initially on the limit cycle. If a single stimulus is given, the trajectory will asymptotically return to the limit cycle, unless the stimulus kicks the state point out of the basin of attraction of the limit cycle (for the FHN system we consider here, this would require the state point to be kicked precisely onto the unstable fixed point enclosed by the limit cycle). However, if one compares the evolution of the oscillator to what it would have been in the absence of perturbation, there will generically be a temporal shift $\Delta T$ : the perturbed oscillator will reach a given point of the limit cycle with an advance or a delay compared to the time the unperturbed oscillator would have reached it. Two curves are used to quantify this effect [59].
(1) The phase-resetting curve (PRC) gives the phase shift

$$
\Delta \phi=\Delta T / T_{0},
$$

which is the temporal shift normalized by the intrinsic period, as a function of the phase

$$
\phi=t_{c} / T_{0} \quad(\bmod 1) .
$$

In this expression, the "coupling time" $t_{c}$ is the time of delivery of the stimulus, measured from the moment at which the state point has crossed an arbitrary fiducial point on the limit cycle called "phase zero." For a cell that fires periodically, a common choice of phase zero is a point on the upstroke of the action potential $[6,53,54,56,72-74]$. The temporal shift and the phase shift are by convention positive when the asymptotic effect of the stimulus is to advance the state point along the limit cycle ("phase advance"), and negative for the reverse ("phase delay") (e.g., $[73,75]) . \Delta \phi$ is usually defined modulo 1 , but we prefer not to restrict $\Delta \phi$ to $[0,1)$ here for reasons we explain later.

(2) The phase transition curve (PTC) gives the "new phase"

$$
\phi^{\prime}=\phi+\Delta \phi \quad(\bmod 1)
$$

as a function of the "old phase" $\phi$. As a map from $S^{1}$ to $S^{1}$, the PTC is characterized by its topological degree, which is the net number of times $\phi^{\prime}$ winds around the unit circle while $\phi$ winds around the unit circle once, or the mean slope of the PTC. The topological degree is also referred to as the phase-resetting "type" [59].

Because the effect of the stimulus depends not only on the old phase, but also on the amplitude of the stimulus, there exists one PRC and PTC for each value of the amplitude. The phase shift and the new phase can be measured exactly only an infinite time after the stimulus has been given, since it takes an infinite time for the state point to return to the limit cycle. However, when the limit cycle is sufficiently attracting, it is a good approximation to consider that the state point is back to the limit cycle within a few intrinsic periods following the application of the stimulus or, in systems where there is a distinctive event occurring during the cycle (such as an action potential), within a few occurrences of that event.

\section{Phase-resetting measurement}

For the system we study in this paper, it turns out to be a good approximation to consider that the oscillator has reached its asymptotic new phase by the second action potential following the stimulation (i.e., we obtain virtually the same PRC and PTC if we wait for a third AP). Therefore, we measured $\Delta \phi$ in the following way (Fig. 9).

Starting with initial conditions corresponding to the chosen phase zero $\left(u_{0} \simeq 0.519, v_{0} \simeq-0.0149\right)$, we applied a stimulus at phase $\phi=t_{c} / T_{0} \in[0,1)$. For our Gaussian-shaped stimulus pulse, $t_{c}$ was taken as the time at which the stimulus goes through its maximum. The increment in phase from one trial to the next was at most 0.001 (a smaller increment was used in the regions of phase where the PTC is particularly steep). 


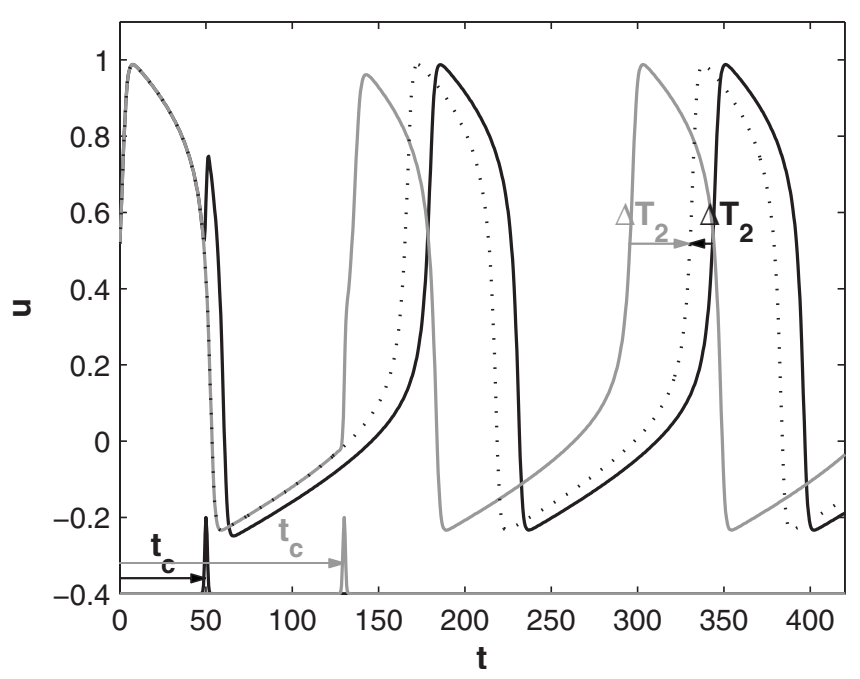

FIG. 9. Phase-shift measurement for two different trials with $I_{0}=0.2$ (solid traces). The dotted trace shows the evolution of the $u$ variable for the unperturbed oscillator. For $t_{c}=50$ (black trace), $\Delta T_{2}<0$ ("phase delay"); for $t_{c}=130$ (gray trace), $\Delta T_{2}>0$ ("phase advance").

Following each stimulation, we waited for the occurrence of two APs and then measured the time $t_{p 2}$ at which the potential $u$ (solid traces in Fig. 9) goes through the "event marker" $\left\{u=u_{0}, d u / d t>0\right\}$ on the second AP. Crossing this event marker is indeed equivalent to crossing phase zero when the state point is on the limit cycle. On the other hand, an unperturbed oscillator (dotted trace in Fig. 9) would have crossed the event marker on the same AP at $t=2 T_{0}$. Therefore, the temporal shift between the two oscillators by the second AP is $\Delta T_{2}=2 T_{0}-t_{p 2}$ and the corresponding phase shift (called the "second transient phase shift" $[73,75,76]$ ) is $\Delta \phi_{2}=\Delta T_{2} / T_{0}$. As we stated above, no significant difference was observed when measuring instead $\Delta T_{3}$ (temporal shift by the third AP), so that we could safely assume $\Delta T \simeq \Delta T_{2}$ and $\Delta \phi \simeq \Delta \phi_{2}$. On the other hand, there were significant differences between $\Delta T_{2}$ and $\Delta T_{1}$ for some combinations of $I_{0}$ and $\phi$.

The criterion we used to acknowledge the occurrence of an AP is that the potential $u$ becomes greater than $u_{\mathrm{AP}}$ $=0.706$, which is the value of the potential at the right knee of the $u$-nullcline of the unforced system [see Fig. 2(b)]. We required additionally that the potential has first to decrease below 0 before a new AP can be elicited, so that deflections occurring during an AP (such as that caused by the stimulus in the black trace in Fig. 9) are not identified as additional APs.

The phase shifts we measure are always smaller than 1 , since the "best" a stimulus of short effective duration can do is to cause the immediate occurrence of one extra action potential. On the other hand, $\Delta \phi$ can take all negative values; in particular it can be quite negative if the stimulus moves the state point close to the unstable fixed point of the system. Therefore we have $\Delta \phi \in(-\infty, 1)$.

Because a stimulus of sufficiently large amplitude is capable not only of advancing or delaying an existing AP, but also of eliciting a new AP [see the first two animations (tem- poral evolution of the potential) in supplementary material [77]], there can be discontinuities of size 1 in the PRC (e.g., in Fig. 10 below) if $\Delta \phi$ is not defined modulo 1. Indeed, for large enough amplitude, there is a critical phase of stimulation such that, for a phase smaller than this value, the perturbation immediately caused by the stimulus in the $u$ wave form does not satisfy the definition of an AP given above, while it does when the phase of stimulation is above the critical value (this critical phase exists no matter the definition of an AP chosen; only its value depends on the definition used). At this threshold value of the phase, a discontinuity appears in the PRC. Its size is effectively 1 provided the state point is back to the limit cycle when the phase-shift measurement is done, which is virtually the case by the second AP here. Such size-1 gaps in the PRC do not make the PTC discontinuous since $\phi^{\prime}$ is defined modulo one. Gaps of size different from 1 appear in the first transient phase shift $\Delta \phi_{1}$, here (not shown) and elsewhere [54,72-76], and lead to discontinuities in the PTC if $\Delta \phi_{1}$ is used to estimate $\Delta \phi$. The reason we wish to keep track of the size-1 gaps in the PRC, by not using the modulo in the definition of $\Delta \phi$, is given in the following section.

\section{Phase-resetting map}

In addition to its intrinsic interest as a characterization of the behavior of an oscillator, the PTC or PRC can be used to derive a one-dimensional (1D) discrete map (the "phaseresetting map") which, under certain conditions, can predict the behavior of the oscillator under periodic forcing (see, e.g., $[6,10,19,52-55])$. Suppose that the $i$ th stimulus is given when the state point of the system lies on the limit cycle, at phase $\phi_{i}$. Suppose also that it takes at most a time $\tau$ for the state point to come back, to within a good approximation (to be defined, but this definition does not matter to the present argument), to the limit cycle after having been perturbed away from it. Then, if the stimulation period $T$ is larger than $\tau$, the state point will be effectively back to the limit cycle when the next stimulus is applied, at phase $\phi_{i+1}$. Calling $\phi_{\tau}$ the phase at which the state point comes back to the limit cycle, we have

$$
\phi_{i+1}=\phi_{\tau}+\frac{T-\tau}{T_{0}}(\bmod 1),
$$

since the state point evolves on the limit cycle from $\phi_{\tau}$ to $\phi_{i+1} . \phi_{\tau}$ is itself related to $\phi_{i}$ by

$$
\phi_{\tau}=\phi^{\prime}\left(\phi_{i}\right)+\frac{\tau}{T_{0}} \quad(\bmod 1),
$$

since $\phi^{\prime}\left(\phi_{i}\right)$ is the asymptotic phase of the oscillator stimulated at $\phi_{i}$ when a hypothetically unperturbed oscillator would cross $\phi_{i}$. So in the end we have

$$
\phi_{i+1}=f\left(\phi_{i}\right)=\phi^{\prime}\left(\phi_{i}\right)+\frac{T}{T_{0}} \quad(\bmod 1),
$$

where $f$ is a one-dimensional circle map (discrete map from $\mathrm{S}^{1}$ to $\left.\mathrm{S}^{1}\right)$ giving the phase just before the $(i+1)$ st stimulus as a function of the phase just before the $i$ th stimulus. It is obtained by simply shifting the PTC vertically by an amount 

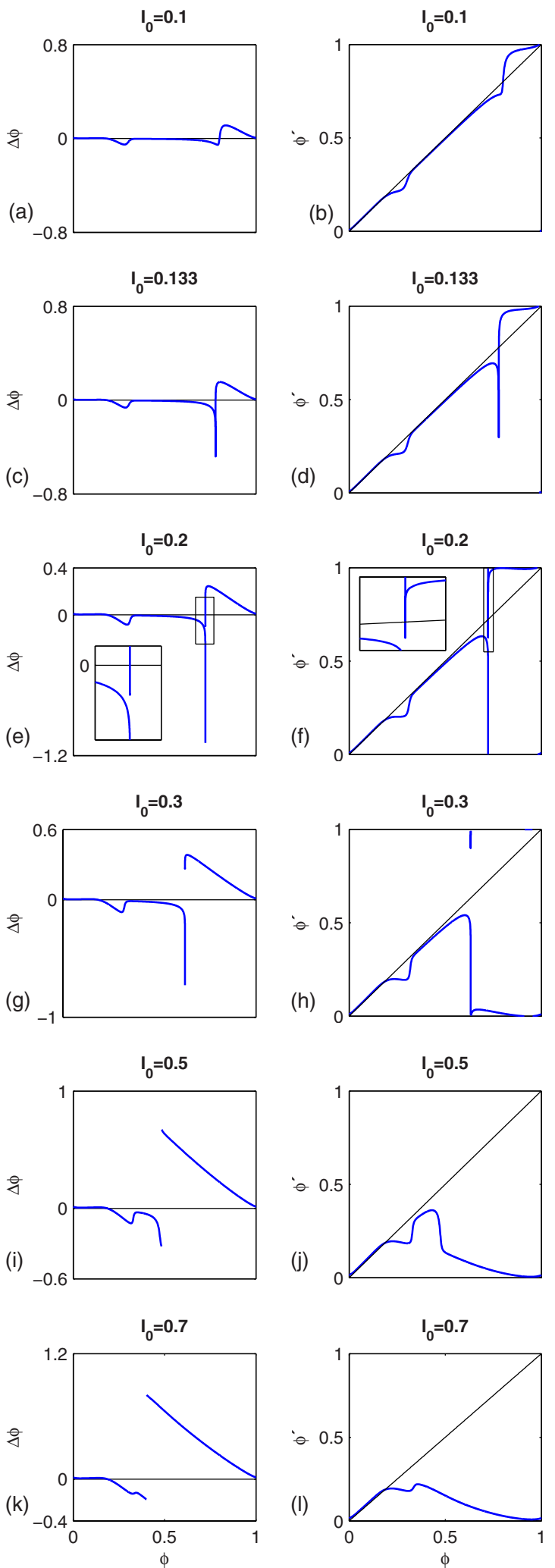

FIG. 10. (Color online) Evolution of the PRC (left) and PTC (right) for increasing stimulation amplitude $I_{0}$. For $I_{0}=0.1$ and 0.133 , the PRC is continuous and the PTC is of degree 1 . For $I_{0}$ $=0.2$ and above, the PRC exhibits a discontinuity of size 1 and the PTC is of degree 0 (zooms help to see this for $I_{0}=0.2$ ). The curves were computed using the direct numerical integration method described in Sec. III A 2.
$T / T_{0}$, and its topological degree is therefore the same as that of the PTC. The fixed points of the $M$ th iterate of the map correspond to period- $M$ solutions for the periodically forced oscillator. They will coincide with solutions of the original ODE (1) when the hypothesis $\tau<T$ is satisfied. One thus expects the map to work better at large stimulation periods. Equivalent ways of formulating the justification for the use of (5) can be found, e.g., in [10,55,58].

An orbit of a circle map can be characterized by its rotation number $\rho$. While this number was originally defined for degree-1 circle maps (see Sec. II B), the definition has been generalized to circle maps of any topological degree [61]. (Note that this is different from generalizing the rotation number to 2D maps, as is done, e.g., in [32].) Assume the circle map $f$ is defined by the restriction to the circle $\mathrm{S}$ of a map $F: \mathbb{R} \rightarrow \mathbb{R}$, that is,

$$
\phi_{i}=f\left(\phi_{i-1}\right)=F\left(\phi_{i-1}\right) \quad(\bmod 1),
$$

for $i \geqslant 1$. We use here $F(x)=x+\Delta \phi(x \bmod 1)+T / T_{0}$. The rotation number for an initial condition $\phi_{0}$ is defined by

$$
\rho\left(f, \phi_{0}\right)=\limsup _{n \rightarrow \infty} \frac{1}{n} \sum_{j=0}^{n} \Delta_{j}\left(\phi_{j}\right)
$$

where

$$
\Delta_{i-1}\left(\phi_{i-1}\right)=F\left(\phi_{i-1}\right)-\phi_{i-1} .
$$

For a periodic orbit of period $M,\left\{\phi_{0}^{*}, \phi_{1}^{*}, \ldots, \phi_{M}^{*}\right\}$ with $\phi_{i}^{*}$ $=f\left(\phi_{i-1}^{*}\right)$ and $\phi_{M}^{*}=\phi_{0}^{*}$, the rotation number is the rational number $\rho=N / M$ where

$$
N=\sum_{j=0}^{M-1} \Delta_{j}\left(\phi_{j}^{*}\right) .
$$

The way we have defined $\Delta \phi$ and $F$ ensures that the critical phases at which, in the PRCs, an extra action potential is generated, are preserved in $F$. This is important because it guarantees that the changes of rotation number that can occur along solution branches will be linked to our definition of an action potential, instead of being completely arbitrary. For instance, it guarantees that the stimulation period $T / T_{0}$ at which, at large stimulation amplitudes, the 1:1 rhythm changes into the 1:2 rhythm along the period-1 branch (Fig. 11), will correspond to the generation of an extra action potential according to our definition. If we had used instead $F(x)=\phi^{\prime}(x \bmod 1)+T / T_{0}$, that stimulation period would have simply been $T / T_{0}=1$, which is meaningless. Use of the last-mentioned $F(x)$, or adding the modulo in the definition of $\Delta \phi$ [Eq. (3)], is thus equivalent to erasing existing information (the size-1 gaps in the PRC) and then artificially reconstructing it.

\section{B. Results}

\section{Phase-resetting and phase transition curves}

Figure 10 shows the evolution of the PRC and PTC for increasing stimulation amplitude, computed by the direct numerical integration method described in Sec. III A 2. For $I_{0}$ 

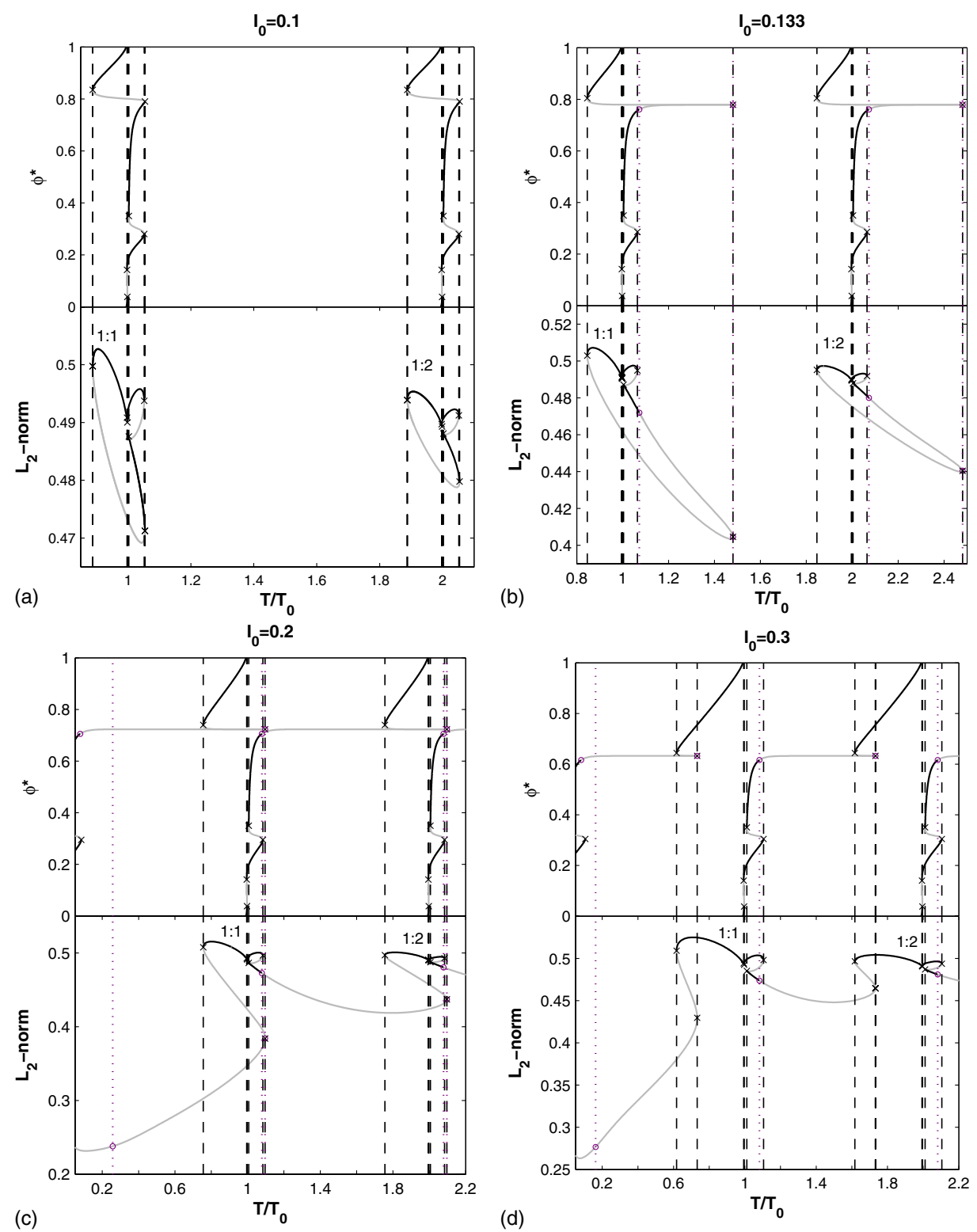

FIG. 11. (Color online) Comparison of the period-1 solutions for the map and for the original ODE (upper and lower sets of curves in each panel) for the values of $I_{0}$ used in Fig. 10. The locations of the bifurcations in the ODE are highlighted by vertical dashed (SN) or dotted (PD) lines. These lines look thick when two bifurcations occur for very close values of $T / T_{0}$.

(d)
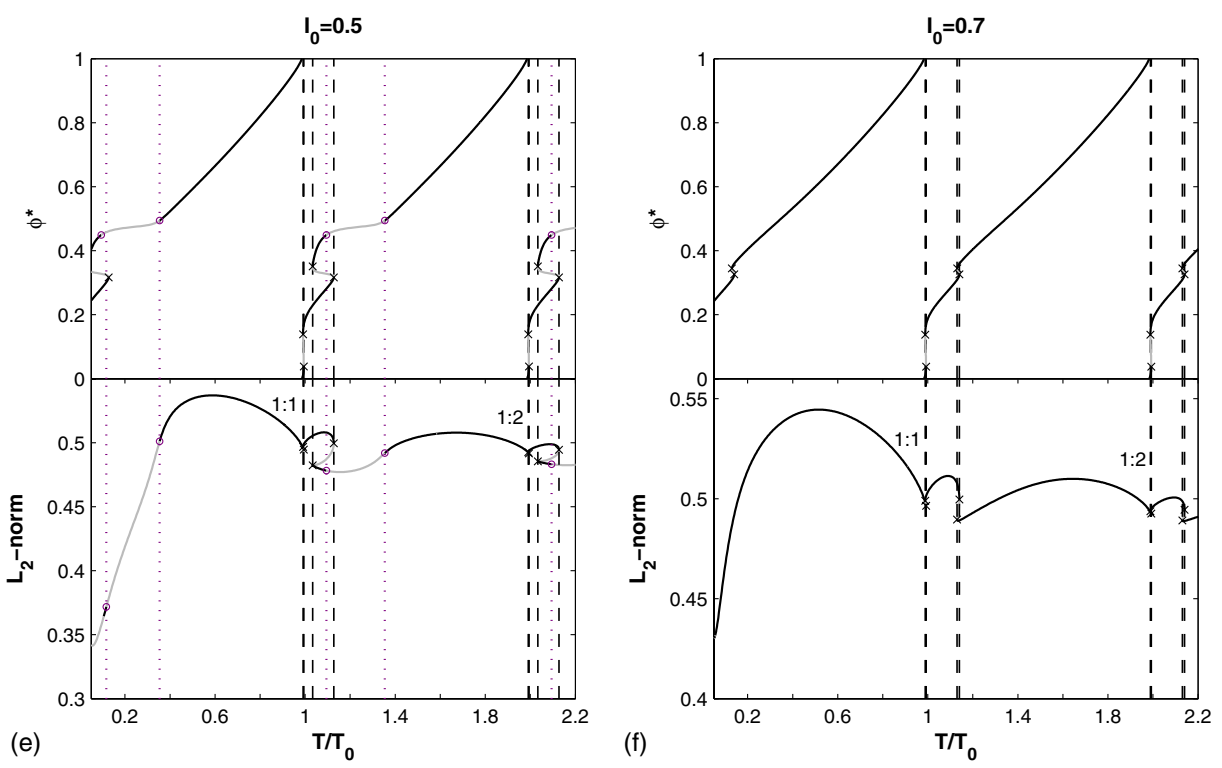
$=0.1$ and 0.133, the PRC is continuous and the PTC has topological degree 1 . For $I_{0}=0.135$, the steepness of the curves has become so large around $\phi \sim 0.78$ that we could not determine their topology even by reducing the step in $\phi$ down to the minimum that double numerical precision allows ( $\simeq 15$ significant decimal digits). That is why we do not show the PRC and PTC for this amplitude in Fig. 10. To get round this difficulty, we used a method originally developed for the computation of (un)stable manifolds of vector fields [78]: we performed continuation of trajectories, defined as the solutions of a boundary-value problem where the initial conditions are fixed to phase zero and the integration time is set to $3 T_{0}$, using the phase $\phi$ of the (single) stimulus given as the main continuation parameter. Because AUTO uses pseudoarclength continuation (which implies including the state variables in addition to the main parameter in the definition of the step), the continuation step size reflects the change of the entire computed trajectory, and not only the change of the main parameter (as is the case of the step size in the direct numerical integration method used above). Therefore, we could follow much more finely, within the limits of double numerical precision, the evolution of the trajectories in the steeply changing region of the PTC. We could then deduce from the observed evolution that the topological degree is 0 at $I_{0}=0.135$. By repeating the procedure with different values of $I_{0}$, we could show that the change of topological degree occurs for $I_{0}=I_{\text {th }}=0.1338 \pm 0.0001$, which is also the amplitude at which the merging of the period-1 loops with the everywhere-unstable period- 1 branch takes place in the bifurcation diagram of the original ODE. We explain the reasons for this coincidence in Sec. III B 4. In the supplementary material [77], two animations made from the trajectories computed by continuation provide a nice way to see that the PTC is degree 1 at $I_{0}=0.133$ and degree 0 at $I_{0}=0.135$ : they show the evolution in the phase plane of the point corresponding to $\phi^{\prime}$ (approximated by the point reached at $t=t_{c}$ $+2 T_{0}$ ) as the point corresponding to $\phi$ winds around the limit cycle once. They also reveal that the change of topological degree in the PTC of a relaxation oscillator involves canardlike trajectories: for $\phi$ in the steep region of the PTC, the trajectories hug the middle branch of the $u$-nullcline along a part of their course.

For all amplitudes above $I_{0}=0.135$ in Fig. 10, the PTC remains degree 0 . Correspondingly, the PRC exhibits a discontinuity of size 1 , reflecting the fact that additional action potentials can be elicited for $I_{0}>I_{\text {th }}$ (cf. the discussion in Sec. III A 2).

\section{Qualitative features of the phase-resetting map}

Because the phase-resetting map is a simple vertical shift of the PTC, a few qualitative characteristics of the bifurcation diagrams for the $1 \mathrm{D}$ map can be deduced directly from the appearance of the PTCs in Fig. 10. For $I_{0}=0.1$, the PTC is monotonic, so that no iterate of the 1D map can exhibit PD points. This is consistent with the bifurcation diagram of the original ODE (Fig. 1), where none of the period- $M$ loops exhibits PD points. In addition, the PTC exhibits two downward deviations from the line of identity. This implies that there will be bistability between period-1 fixed points in the map over a range of $T / T_{0}$ values (range over which the vertical shift of the PTC has four intersections with the line of identity). The corresponding range of $1: 1$ self-bistability is found in the bifurcation diagram of the ODE (Fig. 1).

For $I_{0}=0.133$, the PTC is no longer monotonic, exhibiting regions with slope $\leqslant-1$. This implies that even the first iterate of the 1D map (the "period-1 map") will have PD points. This is consistent with the fact that even the period-1 loops exhibit PD points in Fig. 5.

From $I_{0}=0.2$ to 0.7 in Fig. 10, the PTC gradually flattens out, so that the ranges of $T / T_{0}$ over which the fixed points of the map are unstable gradually shorten. This agrees with the fact that the period- 1 branch becomes stable over increasingly large ranges of $T / T_{0}$ as $I_{0}$ increases in the ODE (Fig. 8).

\section{Period-1 map: Fixed points and their stability}

In order to compare quantitatively the predictions of the phase-resetting map with the results obtained for the original ODE, one needs to compute the fixed points of the map as a function of the stimulation period $T / T_{0}$. In general, this requires solving a nonlinear algebraic equation for each value of the parameter, and continuation methods are often helpful in that process. However, for the kind of map we consider in this paper, where the parameter $T / T_{0}$ appears in an additive fashion only, no computation is needed to determine the curves of fixed points for the period-1 map (first iterate), as we show below.

From (4) and (5), the period-1 map is

$$
\phi_{i+1}=\phi_{i}+\Delta \phi\left(\phi_{i}\right)+T / T_{0} \quad(\bmod 1),
$$

so that its fixed points $\phi^{*}$ satisfy

$$
\Delta \phi\left(\phi^{*}\right)+T / T_{0} \quad(\bmod 1) \quad=0
$$

or

$$
\phi^{*}=(\Delta \phi)^{-1}\left[-T / T_{0} \quad(\bmod 1)\right] .
$$

Of course, for some values of $T / T_{0}$, the inverse function $(\Delta \phi)^{-1}$ is not defined, or is multivalued, but (7) still provides a simple graphical way to obtain $\phi^{*}$ as a function of $T / T_{0}$. Indeed, (7) implies that one simply needs to rotate the PRC by $90^{\circ}$ counterclockwise about the origin, and replicate the curve thus obtained at all distances equal to an integer along the $T / T_{0}$ axis. Computations are needed only to locate the bifurcations along the curves of fixed points. The result of these operations for the six values of $I_{0}$ from Fig. 10 are shown in the upper parts of the panels of Fig. 11. The lower parts of the panels show the corresponding bifurcation diagrams for the original ODE restricted to the period- 1 solutions. Vertical dashed and dotted lines highlight, respectively, the locations of the SN and PD bifurcations for the ODE, in order to facilitate visual comparison with the bifurcations for the map (which are only indicated by the usual crosses and circles).

Quantitative comparison confirms that the phase-resetting map does an excellent job in accounting for the period-1 bifurcations occurring in the ODE until rather small $T / T_{0}$. For example, at $I_{0}=0.5$, the PD point occurring at $T / T_{0}$ 
$=0.353777$ in the ODE coincides with that found in the map up to the first six digits. The phase-resetting map does not however account for the everywhere-unstable period-1 branch existing at small stimulation amplitudes (Figs. 1 and 5). This is because, for solutions belonging to this branch, the state point never reaches the limit cycle of the unforced system (Fig. 3), so that the phase of stimulation is not even defined.

While the bifurcation diagrams in Fig. 11 allow a direct comparison between the two approaches insofar as the locations of the bifurcations are concerned, they do not allow comparison of all the solutions along the branches since the variables for the map and for the ODE are different. In order to make such a comparison, one would need to identify the phase of stimulation for the period-1 solutions of the ODE. The paradox is, however, that such an identification makes sense only when the map is a good approximation of the ODE, since phases are defined only for points belonging to the limit cycle of the unforced system. Nevertheless, one can always identify the point on the limit cycle closest to the state point at the moment when the stimulus is given, compute the phase corresponding to this point, and see how this phase compares with the phase predicted by the 1D map for the same stimulation period. Strictly speaking, this procedure does not yield a comparison between the two approaches (it is only a genuine comparison when the predictions of two approaches coincide, in which case it is pointless), but it does allow one to estimate the value of $T / T_{0}$ at which the $1 \mathrm{D}$ map approximation breaks down.

We have applied this procedure to a sample of the periodic solutions from the left part of the period-1 branch for $I_{0}=0.2\left[T / T_{0}<1.1\right.$ in Fig. 8(a) $]$. The moment at which the stimulus starts is assumed to be $t_{m 3}=t_{\mathrm{st}}-3 \sigma$, where $t_{\mathrm{st}}$ is the time at which the Gaussian-shaped stimulus goes through its maximum, since the effect of the stimulus can still be considered to be negligible before $t=t_{m 3}$. The coordinates of the state point at that moment are then compared to those of a stored array of points on the limit cycle (obtained via a preliminary integration) to determine which of these points is the closest to the state point. We use a weighted Euclidean distance as our metric, with a ten times larger weight for the $v$ variable since it varies over a range about ten times smaller than the $u$ variable. The phase $\phi_{m 3}$ of the closest point on the limit cycle is then identified (this is straightforward provided the preliminary integration is started at phase zero, and that time is stored at each step in addition to the coordinates of the points). We then compare the phase $\hat{\phi}=\phi_{m 3}+3 \sigma / T_{0}$ (mod 1$)$ to the phase $\phi^{*}$ predicted by the map, since the latter is defined considering the time at which the Gaussianshaped stimulus goes through its maximum. This method has been used previously as an alternative to the method described in Sec. III A 2 to determine the PTC [7].

Figure 12(a) shows the projections onto the $(u, v)$ phase plane of the period-1 solutions for the ODE, labeled by a number that increases as $T / T_{0}$ decreases (the solutions numbered 21,23 , and 25 are not shown for the sake of clarity). The circle symbol on each trajectory represents the coordinates of the state point at $t=t_{m 3}$ and the corresponding cross represents the closest point on the limit cycle [the crosses (circles) corresponding to the solutions numbered 7-22 ap-
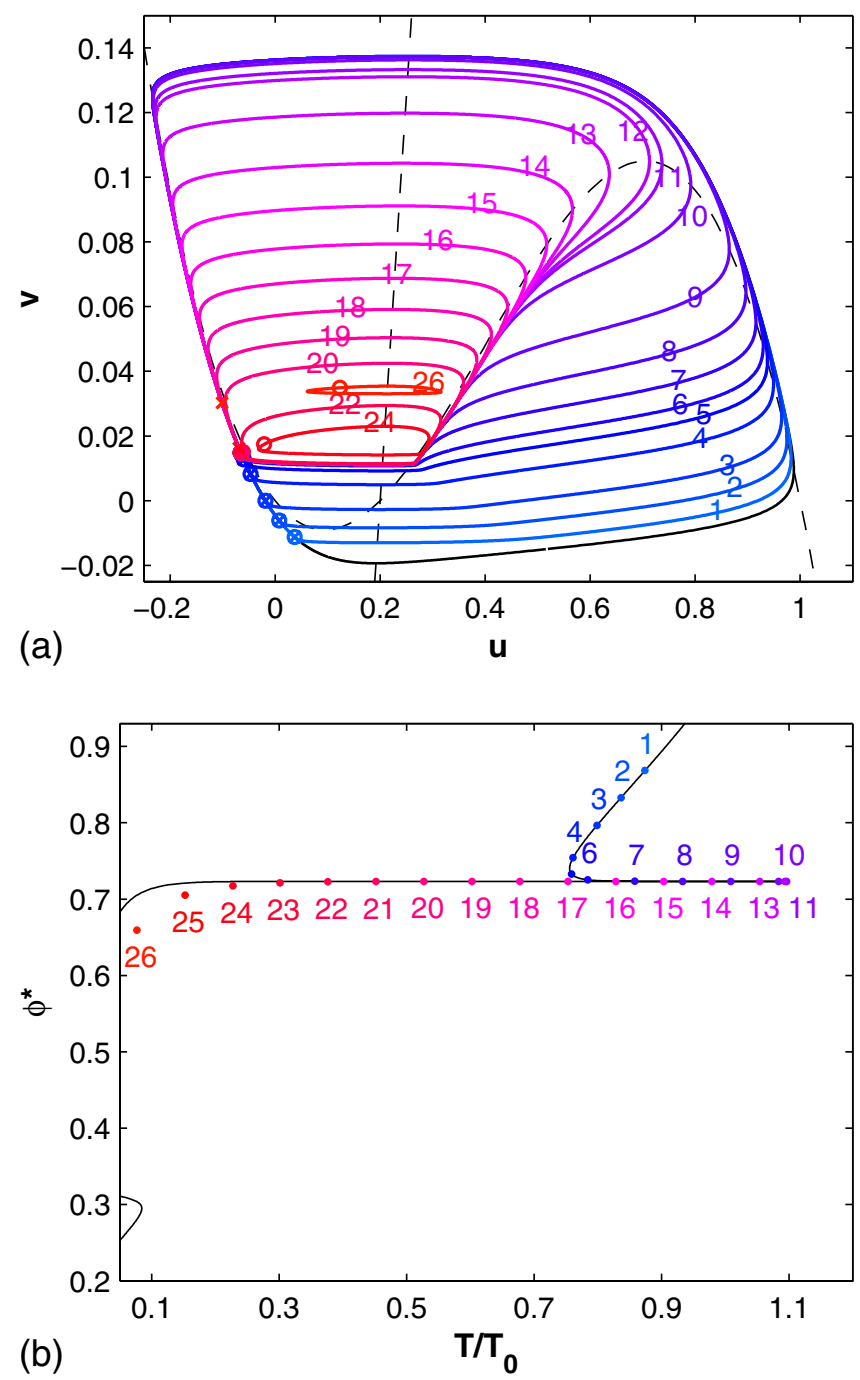

FIG. 12. (Color online) (a) projections onto the phase plane of a sample of period-1 solutions of the ODE for $I_{0}=0.2\left(0.05<T / T_{0}\right.$ $<1.1$ ). The unlabeled solid black curve is the limit cycle of the unforced system, the dashed curves are the nullclines, and the numbered colored curves are the periodic solutions of the ODE. See text for details. (b) phases computed for the solutions shown in (a) (numbered colored dots) and phase predicted by the corresponding period-1 map (solid black curve).

pear superimposed in Fig. 12(a) because they correspond to very close stimulus phases; see Fig. 12(b)]. Figure 12(b) shows the phases computed for the solutions in Fig. 12(a) (numbered colored dots), superimposed on the phases predicted by the map (solid black curve). The two sets of phases start to diverge around $T / T_{0}=0.3$. The discrepancy involves, as expected, the solutions with number 24 and above, since these are the solutions in Fig. 12(a) for which the circles and crosses do not coincide, i.e., solutions for which the assignment of a phase does not make sense. Also nicely illustrated in Fig. 12(a) is the canardlike behavior of most of the unstable limit cycles along this branch (only the periodic solutions labeled 1 to 4 , which are stable, and the periodic solution labeled 26, which is unstable, do not hug the middle branch of the $u$-nullcline along a part of their course). 


\section{Period-1 map: Topology of solution branches}

It is not a coincidence that the topological degree of the PTC changes at the same amplitude $I_{\text {th }}$ as that at which the period-1 loops merge with the everywhere-unstable period-1 branch in the bifurcation diagram of the original ODE. Indeed, Eq. (7) implies that the fixed points of the period-1 map belong to isolated loops when the PTC is of degree 1 and to a unique branch when the PTC is of degree 0 , as we explain now. Since $\Delta \phi=\phi^{\prime}-\phi$, the mean slope of the PRC is obtained by subtracting one from the mean slope of the PTC. Thus, when the PTC is of degree 1, the PRC has a mean slope equal to 0 . Rotating the PRC by $90^{\circ}$ counterclockwise, to obtain the period-1 fixed points, gives a curve with infinite mean slope, that is, a deformation of a vertical line. The ends of this curve, $\phi=0$ and $\phi=1$, thus have the same abscissa $T / T_{0}$. Because $\phi=0$ is the same phase as $\phi=1$, the curve is actually a closed loop. The bifurcation diagram for the period-1 map is thus made up of the replication of the same loop every unit along the $T / T_{0}$ axis [e.g., upper curves in Figs. 11(a) and 11(b)]. In contrast, when the map is of degree 0 , the PRC has a mean slope equal to -1 , which implies that the curve of period-1 fixed points has a mean slope equal to 1. Its ends $\phi=0$ and $\phi=1$ thus span a distance of 1 on the $T / T_{0}$ axis, so that the replication of the curve every unit of $T / T_{0}$ gives in this case a unique continuous branch [e.g., upper curves in Figs. 11(c)-11(f)]. Hence, the bifurcation diagram of any circle map of the form (5) has its period-1 fixed points belonging to isolated loops when the topological degree of the map is one, while they belong to a unique branch when the degree is zero. To our knowledge, this topological property of circle maps had not been underscored before.

As a consequence, if the 1D map is a good approximation of the original ODE, one would expect a change in degree in the map at the same stimulation amplitude as that at which the period-1 loops in the bifurcation diagram of the original ODE merge with the everywhere-unstable period-1 branch, which indeed is what happens. However, it is important to remember that the $1 \mathrm{D}$ map does not account for the everywhere-unstable period-1 branch that exists at small stimulation amplitudes in the original ODE, and whose presence is crucial to the merging phenomenon: from a topological point of view, the period-1 loops could not form an unbounded period-1 branch for $I_{0}>I_{\text {th }}$ if they were not to collide with an unbounded period-1 branch.

The important role of the everywhere-unstable period-1 branch in the main topological change of the bifurcation diagram of the ODE is not surprising to the extent that it reflects the existence of the unstable fixed point of the unforced system. Indeed, for planar oscillators described by a stable limit cycle surrounding a single unstable fixed point, the fixed point is known to be crucially implicated in the change of topological degree of the PTC. In the case of a stimulus of finite duration, the change of topological degree occurs when the "shifted cycle," the locus of the state reached at the end of the stimulus by all the points belonging initially to the limit cycle, intersects the unstable fixed point [79]. However, the notion of a shifted cycle is not really defined for a stimulus with no clear end such as the one we use in this paper.

\section{Period-M maps and translational symmetry of the bifurcation diagram}

The translational symmetry that characterizes the bifurcation diagram for the fixed points of the period-1 map actually extends to the fixed points of all iterates (period- $M$ maps), due to the additive dependence of the map on the bifurcation parameter $T / T_{0}$ [Eq. (5)]. Physically, this corresponds to the fact that stimulating the oscillator at time $t$ or at time $t+T_{0}$ does not make any difference when the state point lies on the limit cycle, since the phase of stimulation will be the same. More precisely, given a circle map of the form (5), if $\left\{\phi_{1}^{*}, \ldots, \phi_{M}^{*}\right\}$ is a period- $M$ orbit for $T=T^{*}$ with rotation number $\rho=N / M$, then $\left\{\phi_{1}^{*}, \ldots, \phi_{M}^{*}\right\}$ is also a period- $M$ orbit for $T=T^{*}+K T_{0}$, where $K$ is any positive integer, and its rotation number is $\rho=(N+K M) / M[19,61]$.

As a consequence, and because we know that the phaseresetting map accounts almost perfectly for the solutions of the ODE at large stimulation periods, one way to evaluate how the map succeeds in approximating the original ODE at small stimulation periods is to determine to what extent the translational invariance is present in the bifurcation diagrams for the ODE. This indirect method is the only procedure we will use to compare the period- $M$ orbits of the map to the period- $M$ solutions of the ODE for $M>1$, i.e., we will not explicitly compute fixed points of the period- $M$ maps.

The translational invariance predicted by the map is well verified in the bifurcation diagram of the ODE for $I_{0}=0.1$ (Fig. 4), since even the bifurcations on the 3:1 loop coincide visually with those of the 3:4 loop, when the later are shifted by -1 (the left $\mathrm{SN}$ is at $T / T_{0}=0.307797$ for the 3:1 loop and at $T / T_{0}=0.307795$ for the 3:4 loop, while the locations of the right $\mathrm{SNs}$ coincide up to 6 digits). For $I_{0}=0.133$ (Fig. 13), however, a large discrepancy is present at the right of the $3: 1 / 3: 4$ loops, whereas the left SNs still coincide up to six decimal digits. This might seem surprising, since one expects a priori the 1D map approximation to break down at the smallest stimulation periods, where the state point gets the smallest amount of time to recover back to the limit cycle following a perturbation. However, at the right end of the 3:1 loop, the stimulus falls at just the right phase to kick the state point near to the fixed point of the unforced system, where the dynamics is very slow. Thus, even if there is more time to recover than at smaller stimulation periods, there is still not enough time to allow the state-point to return to the limit cycle before the next stimulus occurs - hence the 1D map approximation breaks down.

The picture for $I_{0}=0.135$ is even more striking [Figs. 14(a) and 14(b)], with the 3:4 loop being completely different from the 3:1 loop [Fig. 14(a)], and the 2:3 having become a branch while the $2: 1$ is still a loop [Fig. 14(b)]. The phenomenon does not occur for $I_{0}=0.1$ (Fig. 4) because the stimulation amplitude is not large enough to kick the state point from the limit cycle to near the fixed point, and it does not happen for $I_{0}=0.5$ [Figs. 14(e) and 14(f)] because the stimulation amplitude is too large to do so. For $I_{0}=0.2$ [Figs. 14(c) and 14(d)], a small discrepancy is still present at the right end of the period-3 loops, but it is much smaller than the discrepancy at the left end. 


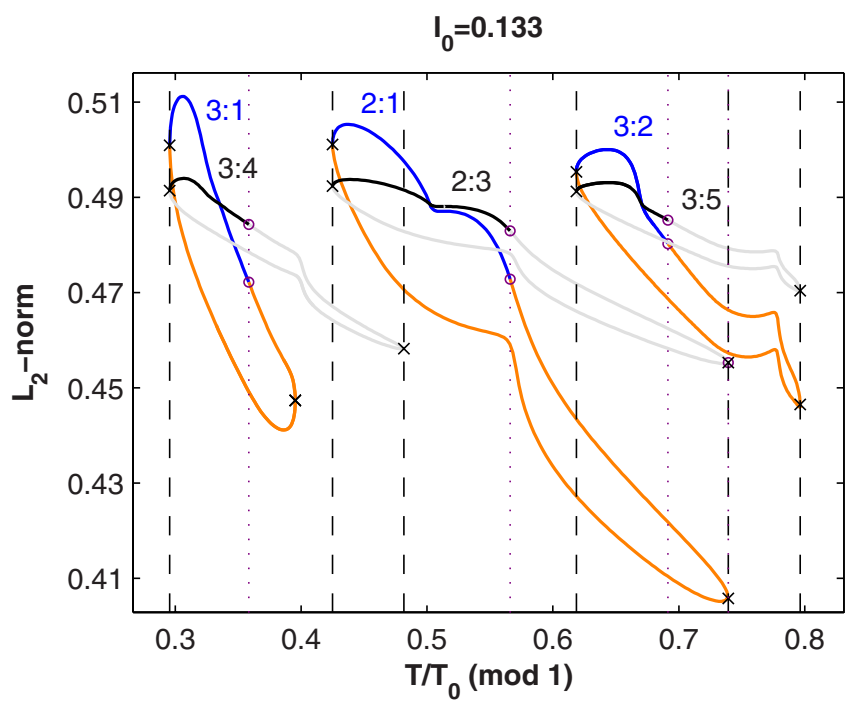

FIG. 13. (Color online) Evaluation of the translational symmetry in the bifurcation diagram of the original ODE for $I_{0}=0.133$, using the procedure and conventions described in Fig. 4. Here, in addition, vertical dotted lines highlight the locations of the PD points for the shifted loops. The period-1 loops are not shown since their symmetry under translation can be deduced from the comparison with the fixed points of the period-1 map (Fig. 11).

\section{DISCUSSION}

\section{A. Continuation applied to pulse-forced oscillators in relaxation regime}

In electrophysiology, one is often interested in relaxation oscillators forced with a periodic train of pulsatile stimuli, the duration of each stimulus being considerably shorter than the intrinsic period. However, the vast bulk of the work on forced oscillators has been for sinusoidally forced systems, and such forcing can lead to behaviors that are qualitatively different from those seen with pulsatile forcing: e.g., with sinusoidal forcing one can have bursting rhythms that one does not see with pulsatile forcing, in which several action potentials ride on the crest of each cycle of the sinusoidal input (see, e.g., Fig. 16 of [39]). In addition, many of these studies on sinusoidal forcing have been in the nonrelaxation regime of the oscillator.

One major advantage of continuation methods is that unstable orbits, as well as stable ones, can be tracked. While there have been a few studies using continuation techniques to investigate pulsatile forcing in the nonrelaxation regime $[29,32,37]$ and one study on sinusoidal forcing in the relaxation regime [13], we are not aware of any continuation analyses on pulsatile forcing in the relaxation regime. In contrast with the aforementioned studies, we have applied continuation to the ODE itself, not to a stroboscopic map of it, in order to benefit from existing functionalities of the AUTO continuation software. However, while it is rather straightforward to run AUTO on sinusoidally forced systems (see the demo "frc" in the AUTO manual [62]), this is not true for most other functional forms of forcing. We have used a recently described method that allows one to use AUTO to study an ODE in which the forcing term is not expressed in terms of sines and cosines, necessitating only minor modifications of the AUTO source code [48]. This method is similar in spirit to one used previously to study stroboscopic maps of forced oscillators (e.g., [23]), in that it takes advantage of the fact that the period of any periodic orbit has to be an integer multiple of the forcing period.

Had we not been led by the results obtained via continuation methods in the ODE, we would probably not have noticed the topological property of the bifurcation diagrams of circle maps which constitutes one of the main findings of this paper.

\section{B. The topology of phase resetting}

As $I_{0}$ is increased, we find a transition from a degree- 1 invertible PTC to a degree- 1 noninvertible PTC to a degree-0 PTC (Fig. 10; see also [9,55]). All these three types of PTC have been reported previously in the FHN oscillator $[6-8,10]$.

A simple continuity argument shows that at a sufficiently low stimulation amplitude, the PTC has to be of degree one and invertible, while at a sufficiently high stimulation amplitude, it has to be of degree zero [59]. A degree-1 curve can be invertible or not, while a degree- 0 curve is, by definition, noninvertible. In models that are intrinsically discontinuous, as stimulation amplitude is increased, there can be a direct transition from a degree-1 invertible PTC to a degree-0 PTC (e.g., $[19,26,34,80,81])$. In continuous models, there is often a transition from a degree-1 invertible curve to a degree-1 non-invertible curve, and then to a degree-0 curve $[9,14,58,73]$. There are also several other studies demonstrating parts of this sequence: e.g., the transition from invertibility to noninvertibility of a degree-1 PTC [54] or from a degree-1 noninvertible to a degree- 0 PTC $[26,30]$. There have been very few systematic studies on noninvertible degree-1 PTCs. In one experimental study, the PTC was found to be noninvertible over about 50\% of the range of amplitude over which it was of degree 1 [54], which is a larger range than we find above (at most 25\%).

Even at the lowest $I_{0}$, there are three bumps in the PRC of our oscillator. For example, at $I_{0}=0.1$ [Fig. 10(a)], the first bump (at $\phi=0.2-0.3$ ) is caused by the stimulus extending the duration of the action potential [as can be seen, e.g., in the black trace in Fig. 2(a)]. The second bump, at $\phi$ $=0.7-0.8$ for $I_{0}=0.1$ [Fig. 10(a)], is due to a prolongation of the "diastolic interval," which is the time between the end of the action potential and the start of the subsequent action potential [gray trace in Fig. 2(a)]. The third bump is at $\phi$ $=0.8-1.0$ and is caused by a shortening of the diastolic interval by the stimulus (the gray trace in Fig. 9 shows this effect for $I_{0}=0.2$ ). While there are cases in experimental (e.g., [54,72]) and modeling [73-75] work on cardiac oscillators where one can see all three of these bumps in the same PRC, the amplitude of the first bump is usually considerably less pronounced than in the FHN oscillator. As we discuss below, the amplitude of this bump has an important consequence in determining whether or not 1:1 self-bistability will be observable.

\section{Discontinuities in the PTC}

We have mentioned above (Sec. III A 2) that an artifactual discontinuity can arise in the PTC if the oscillator is not 

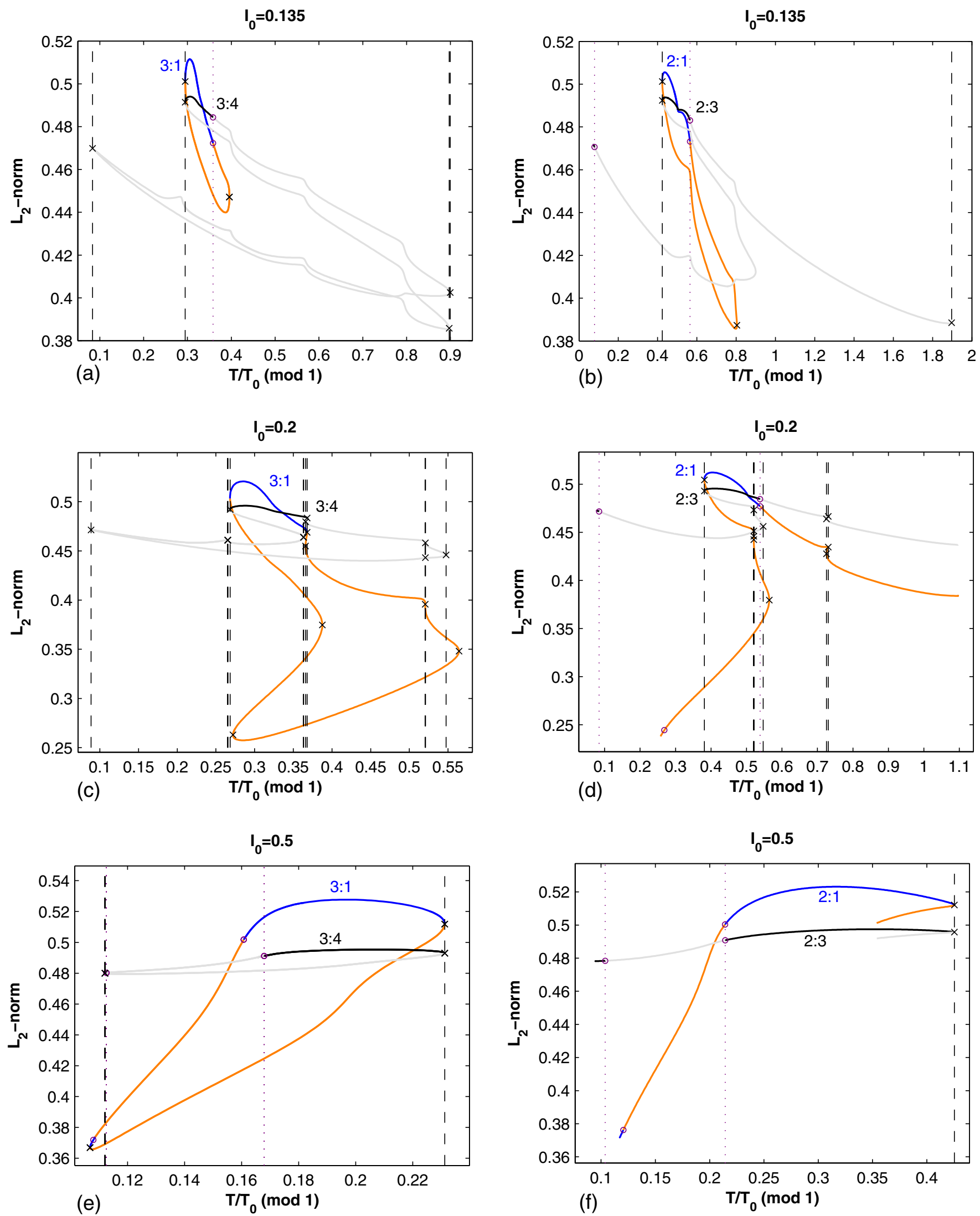

FIG. 14. (Color online) Evaluation of the translational symmetry in the bifurcation diagram of the original ODE for $I_{0}=0.135,0.2$, and 0.5. See Figs. 4 and 13 for the conventions. The 3:1 and 3:4 solutions (left panels) are plotted separately from the 2:1 and 2:3 solutions (right panels) to allow distinguishing the different branches. 
given enough time to recover from the stimulation before making the phase-resetting measurement $[73,76]$. In experimental work on phase resetting in electrophysiological systems, one often encouters a second type of discontinuity in the PTC, with the sequence of transitions for increasing amplitude being: degree-1 invertible $\rightarrow$ degree- 1 noninvertible $\rightarrow$ discontinuous $\rightarrow$ degree- $0[54,72,82]$. The discontinuity is observed in the critical region of phases where the transition from phase delay to phase advance occurs, and is due to the "all-or-none" character of voltage depolarization in many excitable cells.

In 2D differential models where the oscillation is described by a globally attracting limit cycle surrounding a single unstable fixed point, such as the FHN oscillator above, the PTC must be continuous except at the amplitude at which the topological degree changes [79]. This is a consequence of the fact that solutions of "smooth" (Lipschitz continuous) vector fields depend continuously on the initial conditions. However, numerical integration at standard precision can fail to reveal the continuity of the PTC. We have found it necessary to apply continuation techniques to resolve the apparent discontinuity in the FHN model (Sec. III B 1). While continuation has previously been used in an ionic model to reveal the continuity of the PTC [82], this was done in an indirect way, in that the main continuation parameter was not the phase of stimulation, but rather the value reached by the voltage immediately after the stimulus. This was done to allow continuation on the unforced equations, because the stimulus used (a rectangular pulse) was not $\mathcal{C}^{1}$ and thus not suitable for continuation. The method we employ above uses the phase of stimulation $\phi$ itself as the main continuation parameter, and so allows a direct unambiguous demonstration of the continuity of the PTC. Oscillator models that are formulated in an inherently discontinuous fashion (e.g., integrate-and-fire models $[49,60,79])$ exhibit unavoidable discontinuities in their PTCs.

\section{1:1 self-bistability}

Bistability and the resultant hysteresis have been well known in forced relaxation oscillators for a very long time (e.g., $[12,83])$. We observe several forms of bistability in the forced FHN oscillator, among which are $1: 1 \leftrightarrow 1: 1$ (Figs. 1 and $2, I_{0}=0.1$; Fig. 8), $1: 1 \leftrightarrow 2: 1$ (Fig. 8, $I_{0}=0.5$ ), and $2: 1 \leftrightarrow 3: 1$ (Fig. 8, $I_{0}=0.5$ ). We shall restrict the present discussion to only one of these bistabilities: the $1: 1 \leftrightarrow 1: 1$ bistability, or 1:1 self-bistability.

In the forced FHN oscillator, the 1:1 self-bistability extends from zero amplitude all the way up to very high $I_{0}$ (Fig. 8). In the two-parameter plane, this self-bistability corresponds to the presence of 1:1 Arnol'd flames in addition to the main 1:1 SN curve (Fig. 6). The 1:1 self-bistability is accounted for by the phase-resetting map (Fig. 11), with the origin of the main range of bistability being traceable to the fact that there are two regions ("bumps") of phase delay in the PRC (Fig. 10, left). At the lowest amplitudes at which this self-bistability is seen, the map is an invertible degree-1 circle map [e.g., $I_{0}=0.1$, Fig. $\left.10(\mathrm{~b})\right]$, which is in contrast to other forms of (non-self-)bistability, which require nonin- vertibility: i.e., either a noninvertible degree- 1 or a degree- 0 map (e.g., $[9,26,54,61,81,84,85])$. At the largest amplitudes at which we observed the 1:1 self-bistability, the phaseresetting map is a degree- 0 circle map [e.g., $I_{0}=0.7$, Fig. $10(1)$. Recent experimental and modeling work on a hydrodynamical relaxation oscillator [85] confirms the occurrence of 1:1 self-bistability in the degree- 0 regime of phase resetting (it is not clear whether an earlier report on the FHN oscillator was for a degree-0 or a degree-1 noninvertible phase-resetting map (Fig. 5c of [9]).

In most published diagrams of Arnol'd tongues from maps and ODEs, there is an amplitude below which no bistability is seen (e.g., Fig. 78 in [69]; Fig. 5 in [54]; Fig. 8 in [8]). This is rather surprising given that the presence of an Arnol'd flame in at least one of the $N / M$ Arnol'd tongues has been shown to be a generic feature of invertible circle maps, and therefore also of periodically forced planar oscillators, at small forcing amplitude [70]. We see two possible reasons for which self-bistability has rarely been detected.

First, it could be that self-bistability is restricted to very small ranges of parameters. There are indications that this is the case, in particular, for the 1:1 self-bistability. Indeed, in electrophysiological experimental [54,72] and modeling [73-75] work, one can see PTCs with two downward bumps, but the amplitude of the first bump is typically very small. This implies that the range of $T / T_{0}$ over which the $1: 1$ selfbistability would be predicted to exist would be very narrow. In one study where a parametric fit was made to the PRC in order to obtain the phase-resetting map over a wide range of amplitude, this first bump was even completely ignored, and so 1:1 self-bistability, had it been truly present, would not have been predicted to exist [54]. Thus, a very carefully targeted experimental search would have to be made to look for 1:1 self-bistability, which might not be found in the presence of noise.

The second possible reason is that most of the forced oscillators that have been studied hitherto are nongeneric in the sense defined in [70], so that they cannot exhibit selfbistability. This is true, e.g., of the famous canonical "sinemap" model of Arnol'd [69]. In addition, the majority of experimental and modeling studies on forced oscillators used forcing with a pure sine wave, while the few works in which self-bistability has been reported employed a forcing wave form with higher harmonics $[63,86,87]$. This could reflect the fact that the first of these two categories of forced oscillators is nongeneric.

\section{E. The omnipresent period-1 solution in forced oscillators}

In the bifurcation diagram of the ODE, there exists, at sufficiently small $I_{0}$, an everywhere-unstable period- 1 branch (e.g., Figs. 1, 3, and 5), corresponding to the persistence of the unstable period-1 solution enclosed by the invariant torus. The everywhere-unstable period-1 branch merges with the period-1 loops as the amplitude is raised, so that one then has alternating stable and unstable period-1 segments (e.g., Figs. 7 and 8). This evolution of the bifurcation diagram for the period-1 solutions had been predicted analytically many decades ago for the sinusoidally forced van der Pol oscillator, but in the weakly nonlinear limit (see [88] for the origi- 
nal result about the 1:1 solution, and, e.g., $[15,16]$ for the extension to other period-1 solutions). Numerical studies on nonrelaxation oscillators have confirmed this prediction $[23,25]$ and have shown that the collision of the two period-1 solutions corresponds to the breaking of the invariant torus for the corresponding values of $\left(T, I_{0}\right)$. They have also shown that the collision of period- 2 solutions, such as the one that is about to occur for the $2: 1$ rhythm in the bifurcation diagram for $I_{0}=0.135$ (Fig. 7), implies the breaking of the invariant torus at the corresponding parameter values as well. Our numerical results indicate that the same collision scenarios occur in a relaxation oscillator.

The existence of a period-1 solution for all values of $T$ and $I_{0}$ has been proven for the periodically forced FHN oscillator [89], as well as for a simple forced 2D oscillator (the Poincaré oscillator) [34]. However, these two proofs say nothing about the stability of the period-1 solution and the possible coexistence of other period- 1 solutions, whereas the analytical and numerical results discussed above shed light on these aspects.

\section{F. Excitable systems}

There have been many studies on periodically forced excitable systems, in which there is no stable limit cycle present in the unforced system, but only a stable equilibrium point. Several of these studies have been on the original FHN model, where many of the rhythms that we describe above have also been seen $[10,11,38-48]$. Continuation methods have been applied to study the excitable FHN equations, with either sinusoidal forcing [39] or pulsatile forcing [48].

There are various analogs of the PRC that have been used to characterize excitable systems $[10,41,42,46]$. Onedimensional maps can be derived from these curves or from other curves involving parameters such as action potential duration, the latency to an action potential, or the area under the voltage wave form (e.g., [40,43,47]). Return maps of these parameters or of a system variable (e.g., $[40,47])$ can also be obtained. Phenomena such as bistability, perioddoubling bifurcations, and chaos can then be associated with the properties of these 1D maps, which, instead of being circle maps, are noninvertible 1D maps of the interval. Of course, the 1D description breaks down at some point and one then has to resort eventually to a 2D map [11]. It is only in the singular limit that one ends up with a true onedimensional description of the system [14,44-46].

\section{SUMMARY AND PERSPECTIVES}

We have compared the dynamics of a periodically forced relaxation oscillator to that of a one-dimensional discrete map derived from the phase-resetting curve of this oscillator. We have shown that the agreement between the two approaches is, for most stimulation amplitudes, very good until rather small stimulation periods. The discrepancies observed are easily explained in terms of the violation of the hypotheses underlying the derivation of the 1D map. In addition, guided by the topological changes observed in the bifurcation diagram of the original ODE as the stimulation amplitude is raised, we have shown that the generic bifurcation structure of a circle map where the bifurcation parameter appears in an additive fashion only is to have the period-1 fixed points belonging to loops when the map is of topological degree one, and to a unique continuous branch when the topological degree is zero. To our knowledge, this is the first time this property of circle maps has been underscored.

While we have restricted our bifurcation analysis to a few selected values of the stimulation amplitude, it would be interesting to perform a full two-parameter study of the forced relaxation oscillator, i.e., to extend Fig. 6 to other Arnol'd tongues, and, further, to investigate how these tongues evolve as the parameter $\varepsilon$ (which controls the degree of relaxation of the oscillator) is varied. Such a three-parameter bifurcation analysis of a forced oscillator has already been performed [13], but it involved a symmetrical oscillator (the van der Pol oscillator) and sinusoidal forcing. It would thus be interesting to repeat this kind of study for the case of pulsatile forcing and a nonsymmetrical relaxation oscillator.

\section{ACKNOWLEDGMENTS}

H.C. is grateful to the FRIA (Fonds pour la Formation à la Recherche dans l'Industrie et dans l'Agriculture, Belgium) for financial support and to the Centre for Nonlinear Dynamics in Physiology and Medicine, McGill University, for facilitating a visit to Montreal during which part of this work was done.
[1] B. van der Pol, Philos. Mag. 2, 978 (1926).

[2] J. Guckenheimer, K. Hoffman, and W. Weckesser, SIAM J. Appl. Dyn. Syst. 2, 1 (2003).

[3] R. FitzHugh, Biophys. J. 1, 445 (1961).

[4] K. F. Bonhoeffer, J. Gen. Physiol. 32, 69 (1948).

[5] L. Glass and M. C. Mackey, From Clocks to Chaos (Princeton University Press, Princeton, NJ, 1988).

[6] S. Scott, Ph.D. thesis, State University of New York, Buffalo, New York, 1979.

[7] M. R. Guevara, L. Glass, M. C. Mackey, and A. Shrier, IEEE Trans. Syst. Man Cybern. 13, 790 (1983).

[8] T. Nomura, S. Sato, S. Doi, J. P. Segundo, and M. D. Stiber,
Biol. Cybern. 69, 429 (1993).

[9] T. Nomura, S. Sato, S. Doi, J. P. Segundo, and M. D. Stiber, Biol. Cybern. 72, 55 (1994).

[10] K. Yoshino, T. Nomura, K. Pakdaman, and S. Sato, Phys. Rev. E 59, 956 (1999).

[11] A. Rabinovitch and M. Friedman, Phys. Lett. A 355, 319 (2006).

[12] E. A. Jackson, Perspectives of Nonlinear Dynamics (Cambridge University Press, Cambridge, U.K., 1990), Vol. 1.

[13] R. Mettin, U. Parlitz, and W. Lauterborn, Int. J. Bifurcation Chaos Appl. Sci. Eng. 3, 1529 (1993).

[14] E. J. Ding, Phys. Scr. 38, 9 (1988). 
[15] C. Hayashi, H. Shibayama, and Y. Nishikawa, IRE Trans. Circuit Theory 7, 413 (1960).

[16] C. Hayashi, Nonlinear Oscillations in Physical Systems (McGraw-Hill, New York, 1964).

[17] K. Tomita and T. Kai, J. Stat. Phys. 21, 65 (1979).

[18] Y. Ueda and N. Akamatsu, IEEE Trans. Circuits Syst. CAS28, 217 (1981).

[19] M. R. Guevara and L. Glass, J. Math. Biol. 14, 1 (1982).

[20] D. L. Gonzalez and O. Piro, Phys. Rev. Lett. 50, 870 (1983).

[21] H. Kawakami, IEEE Trans. Circuits Syst. CAS-31, 248 (1984).

[22] D. G. Aronson, R. P. McGehee, I. G. Kevrekidis, and R. Aris, Phys. Rev. A 33, 2190 (1986).

[23] I. G. Kevrekidis, L. D. Schmidt, and R. Aris, Chem. Eng. Sci. 41, 1263 (1986).

[24] I. G. Kevrekidis, R. Aris, and L. D. Schmidt, Chem. Eng. Sci. 41, 1549 (1986).

[25] I. G. Kevrekidis, R. Aris, and L. D. Schmidt, Physica D 23, 391 (1986).

[26] E. J. Ding, Phys. Rev. A 35, 2669 (1987).

[27] E. J. Ding, Phys. Rev. A 36, 1488 (1987).

[28] I. G. Kevrekidis, AIChE J. 33, 1850 (1987).

[29] I. Schreiber, M. Dolník, P. Choc, and M. Marek, Phys. Lett. A 128, 66 (1988).

[30] M. Dolník, J. Finkeová, I. Schreiber, and M. Marek, J. Phys. Chem. 93, 2764 (1989).

[31] W. Vance and J. Ross, J. Chem. Phys. 91, 7654 (1989).

[32] B. B. Peckham, Nonlinearity 3, 261 (1990).

[33] C. Knudsen, J. Sturis, and J. S. Thomsen, Phys. Rev. A 44, 3503 (1991).

[34] L. Glass and J. Sun, Phys. Rev. E 50, 5077 (1994).

[35] N. Inaba, R. Fujimoto, H. Kawakami, and T. Yoshinaga, Electron. Commun. Jpn., Part 2: Electron. 83, 35 (2000).

[36] M. Sekikawa, N. Inaba, T. Yoshinaga, and H. Kawakami, Electron. Commun Jpn Part 3: Fundam. Electron. Sci. 87, 30 (2004).

[37] F. Schilder and B. B. Peckham, J. Comput. Phys. 220, 932 (2007).

[38] M. Feingold, D. L. Gonzalez, O. Piro, and H. Viturro, Phys. Rev. A 37, 4060 (1988).

[39] J. C. Alexander, E. S. Doedel, and H. G. Othmer, SIAM J. Appl. Math. 50, 1373 (1990).

[40] S. Sato and S. Doi, Math. Biosci. 112, 243 (1992).

[41] A. Rabinovitch, R. Thieberger, and M. Friedman, Phys. Rev. E 50, 1572 (1994).

[42] S. Doi and S. Sato, Math. Biosci. 125, 229 (1995).

[43] D. T. Kaplan, J. R. Clay, T. Manning, L. Glass, M. R. Guevara, and A. Shrier, Phys. Rev. Lett. 76, 4074 (1996).

[44] M. Xie, H. G. Othmer, and M. Watanabe, Physica D 98, 75 (1996).

[45] H. G. Othmer and M. Xie, J. Math. Biol. 39, 139 (1999).

[46] S. Coombes and A. H. Osbaldestin, Phys. Rev. E 62, 4057 (2000).

[47] E. N. Cytrynbaum, J. Theor. Biol. 229, 69 (2004).

[48] H. Croisier and P. C. Dauby, J. Theor. Biol. 246, 430 (2007).

[49] L. Glass and M. C. Mackey, J. Math. Biol. 7, 339 (1979).

[50] L. Glass and R. Perez, Phys. Rev. Lett. 48, 1772 (1982).

[51] L. Glass and J. Bélair, in Nonlinear Oscillations in Biology and Chemistry, edited by H. Othmer, Lectures Notes in Biomathematics Vol. 66, (Springer-Verlag, Berlin, 1986), pp. 232243.
[52] D. H. Perkel, J. H. Schulman, T. H. Bullock, G. P. Moore, and J. P. Segundo, Science 145, 61 (1964).

[53] M. R. Guevara, L. Glass, and A. Shrier, Science 214, 1350 (1981).

[54] L. Glass, M. R. Guevara, J. Belair, and A. Shrier, Phys. Rev. A 29, 1348 (1984).

[55] A. Campbell, A. Gonzalez, D. L. Gonzalez, O. Piro, and H. A. Larrondo, Physica A 155, 565 (1989).

[56] J. M. Anumonwo, M. Delmar, A. Vinet, D. C. Michaels, and J. Jalife, Circ. Res. 68, 1138 (1991).

[57] M. R. Guevara, A. Shrier, and L. Glass, in Cardiac Electrophysiology: From Cell to Bedside, 1st ed., edited by D. P. Zipes and J. Jalife (Saunders, Philadelphia, 1990), pp. 192201.

[58] G. A. Cecchi, D. L. Gonzalez, M. O. Magnasco, G. B. Mindlin, O. Piro, and A. J. Santillan, Chaos 3, 51 (1993).

[59] A. T. Winfree, The Geometry of Biological Time, 2nd ed. (Springer, New York, 2000).

[60] L. Glass, Nature (London) 410, 277 (2001).

[61] J. Blair and L. Glass, Physica D 16, 143 (1985).

[62] E. J. Doedel, A. R. Champneys, T. F. Fairgrieve, Y. A. Kuznetsov, B. Sandstede, and X. Wang, AUTO97: Continuation and bifurcation software for ordinary differential equations, 1998, http://cmvl.cs.concordia.ca/publications/auto97.ps.gz.

[63] B. B. Peckham and I. G. Kevrekidis, Nonlinearity 15, 405 (2002).

[64] S. Wiggins, Introduction to Applied Nonlinear Dynamical Systems and Chaos (Springer, New-York, 1990).

[65] N. Fenichel, Indiana Univ. Math. J. 21, 193 (1971).

[66] S. Wiggins, Normally Hyperbolic Invariant Manifolds in Dynamical Systems (Springer-Verlag, New York, 1994).

[67] H. Poincaré, Oeuvres I (Gauthier-Villar, Paris, 1928).

[68] G. R. Hall, SIAM J. Math. Anal. 15, 1075 (1984).

[69] V. I. Arnold, Geometrical Methods in the Theory of Ordinary Differential Equations (Springer, New York, 1983).

[70] R. P. McGehee and B. B. Peckham, Int. J. Bifurcation Chaos Appl. Sci. Eng. 6, 315 (1996).

[71] J. Guckenheimer, K. Hoffman, and W. Weckesser, Int. J. Bifurcation Chaos Appl. Sci. Eng. 10, 2669 (2000).

[72] M. R. Guevara, A. Shrier, and Ł. Glass, Am. J. Physiol. 251, H1298 (1986).

[73] M. R. Guevara and H. J. Jongsma, Am. J. Physiol. 258, H734 (1990).

[74] D. G. Tsalikakis, H. G. Zhang, D. I. Fotiadis, G. P. Kremmydas, and L. K. Michalis, Comput. Biol. Med. 37, 8 (2007).

[75] M. R. Guevara and A. Shrier, Biophys. J. 52, 165 (1987).

[76] M. Kawato, J. Math. Biol. 12, 13 (1981).

[77] See EPAPS Document No. E-PLEEE8-78-102812. For more information on EPAPS, see http://www.aip.org/pubservs/ epaps.html.

[78] B. Krauskopf, H. M. Osinga, E. J. Doedel, M. E. Henderson, J. Guckenheimer, A. Vladimirsky, M. Dellnitz, and O. Junge, Int. J. Bifurcation Chaos Appl. Sci. Eng. 15, 763 (2005).

[79] L. Glass and A. T. Winfree, Am. J. Physiol. 246, R251 (1984).

[80] D. L. Gonzalez and O. Piro, Phys. Lett. 101, 455 (1984).

[81] J. P. Keener and L. Glass, J. Math. Biol. 21, 175 (1984).

[82] T. Krogh-Madsen, L. Glass, E. J. Doedel, and M. R. Guevara, J. Theor. Biol. 230, 499 (2004).

[83] B. van der Pol and J. van der Mark, Nature (London) 120, 363 (1927). 
[84] R. Perez and L. Glass, Phys. Lett. 90A, 441 (1982).

[85] H. Gonzalez, H. Arce, and M. R. Guevara, Phys. Rev. E 78, 036217 (2008).

[86] P. Rehmus, W. Vance, and J. Ross, J. Chem. Phys. 80, 3373 (1984).
[87] M. Rivera, P. Parmananda, and M. Eiswirth, Phys. Rev. E 65, 025201(R) (2002).

[88] B. van der Pol, Philos. Mag. 3, 65 (1927).

[89] T. Kostova, R. Ravindran, and M. Schonbek, Int. J. Bifurcation Chaos Appl. Sci. Eng. 14, 913 (2004). 